\title{
ADDITIONAL MATERIALS ON FEATURE ELIMINATION IN KERNEL MACHINES IN MODERATELY HIGH DIMENSIONS
}

\author{
By Sayan Dasgupta, Yair Goldberg, and Michael R. Kosorok
}

S1. Discussion I: Functional spaces on lower dimensional domains. The aim of this section is to provide some results on the restricted spaces defined in Definition 1 in Section 2.3.

S1.1. Further discussions on the lower dimensional spaces $\mathcal{F}^{J}$. We provide a few results here that connect the restricted functional spaces with the original one. In view of Definition 1 , we can define $\mathcal{L}_{\infty}{ }^{J}(\mathcal{X})=\left\{f \circ \pi^{J^{c}}\right.$ : $\left.f \in \mathcal{L}_{\infty}(\mathcal{X})\right\}$. Then Lemma S1 below says that $\left.\mathcal{L}_{\infty}{ }^{J}\left(\mathcal{X}^{J}\right) \equiv \mathcal{L}_{\infty}{ }^{J}(\mathcal{X})\right|_{\mathcal{X}^{J}}$ is isomorphic to the space $\mathcal{L}_{\infty}\left(\mathcal{X}^{J}\right)$. Lemma $\mathrm{S} 2$ below provides some results connecting the original RKHS with its lower dimensional versions. A related lemma, Lemma S3 is also given below, noting similar results for any general space. These results aim to show that many of the nice properties of a given functional space are carried forward to their re-adaptations under Definition 1. We prove Lemma S1 and S3, while the proof for Lemma S2 is omitted as it follows from Lemma S3 trivially.

Lemma S1. $\quad \mathcal{L}_{\infty}^{J}\left(\mathcal{X}^{J}\right)=\mathcal{L}_{\infty}\left(\mathcal{X}^{J}\right)$

Lemma S2. Let $H \subset \mathcal{L}_{\infty}(\mathcal{X})$ be a non-empty $R K H S$ on $\mathcal{X}$. Then for any $J \subset\{1,2, \ldots, d\}$,

1. If $H$ is dense in $\mathcal{L}_{\infty}(\mathcal{X})$, then $H^{J}$ is dense in $\mathcal{L}_{\infty}\left(\mathcal{X}^{J}\right)$.

2. If the $\|\cdot\|_{\infty}$ closure $\overline{B_{H}}$ of the unit ball $B_{H}$ is compact, then so is $\overline{B_{H^{J}}}$.

3. If $H$ is separable, then so is $H^{J}$.

4. $e_{i}\left(i d: H^{J} \mapsto L_{\infty}(\mathcal{X})\right) \leq e_{i}\left(i d: H \mapsto L_{\infty}(\mathcal{X})\right)$, where $e_{i}(i d: H \mapsto$ $\left.L_{\infty}(\mathcal{X})\right)$ is the $i^{\text {th }}$ entropy number of the unit ball $B_{H}$ of the RKHS $H$, with respect to the $\|\cdot\|_{\infty}$-norm.

NOTE: Condition 1 holds true even if $\mathcal{L}_{\infty}(\mathcal{X})$ is replaced by any space that admits the nested structure.

Now we provide Lemma S3 noting similar results for any general space.

Lemma S3. Let $\mathcal{F} \subset \mathcal{L}_{\infty}(\mathcal{X})$ be a non-empty functional subspace. Then for any $J \subseteq\{1,2, \ldots, d\}$, 
1. If $\mathcal{F}$ is dense in $\mathcal{L}_{\infty}(\mathcal{X})$, then $\mathcal{F}^{J}$ is dense in $\mathcal{L}_{\infty}^{J}(\mathcal{X})$.

2. If $\mathcal{F}$ is compact, then so is $\mathcal{F}^{J}$.

3. $e_{i}\left(\mathcal{F}^{J},\|\cdot\|_{\infty}\right) \leq e_{i}\left(\mathcal{F},\|\cdot\|_{\infty}\right), \forall i \geq 1$ where $e_{i}\left(\mathcal{F},\|\cdot\|_{\infty}\right)$ is the $i^{\text {th }}$ entropy number of the set $\mathcal{F}$ with respect to the $\|\cdot\|_{\infty}$-norm as defined in Section 2.6.

NOTE: Condition 1 holds even if $\mathcal{L}_{\infty}(\mathcal{X})$ is replaced by any space that admits the nested structure.

S1.2. RKHS in lower dimensions. Note that in kernel machines, the minimization is computed over an RKHS, and hence, while defining these lower dimensional spaces we need to ensure that these spaces are RKHSs as well. To that effect, we begin this section by providing an alternate way to define the lower dimensional versions of a given RKHS that preserves the reproducing property.

DeFinition S1. For a given RKHS H indexed by a kernel $k$ and a set of indices $J \subseteq\{1,2, . ., d\}$, define $H^{J} \equiv H_{k \circ \pi^{J^{c}}}(\mathcal{X})$, where $k \circ \pi^{J^{c}}(x, y):=$ $k\left(\pi^{J^{c}}(x), \pi^{J^{c}}(y)\right)$.

Note immediately that Definition S1 allows us to create lower dimensional versions of $H$ in a way which ensures that these spaces are RKHSs as well. This inevitably raises questions about the validity of Definition 1 from Section 2.3. However both Definitions 1 and S1 yield the same RKHS space $H^{J}$.

To see this, let $\mathcal{X}_{0}$ be a subset of $\mathcal{X}$ and $k^{(0)}(x, y)$ be the restriction of a kernel $k$ on $\mathcal{X}_{0}$. Then $k^{(0)}(x, y)$ is a valid kernel on $\mathcal{X}_{0}$ by Proposition 5.13 of Paulsen [2009]. Let $H_{k}(\mathcal{X})$ be the RKHS with respect to $k(x, y)$, and $H_{k^{(0)}}(\mathcal{X})$ be the one with respect to $k^{(0)}(x, y)$. Then by Theorem 5.14 of Paulsen [2009], if we define $\varphi$ to be the inclusion id map from $\mathcal{X}_{0}$ to $\mathcal{X}$, we have $H_{k^{(0)}}\left(\mathcal{X}_{0}\right)=\left\{\left.f\right|_{\mathcal{X}_{0}}: f \in H_{k}(\mathcal{X})\right\}$ and $\|g\|_{H_{k}(0)}=\min \left\{\|f\|_{H_{k}}:\left.f\right|_{\mathcal{X}_{0}}=\right.$ $g$ for $g \in H_{k^{(0)}}\left(\mathcal{X}_{0}\right)$. Taking $\mathcal{X}_{0} \equiv \mathcal{X}^{J}$ and $k^{(0)}(x, y) \equiv k\left(\pi^{J^{c}}(x), \pi^{J^{c}}(y)\right)$, we immediately obtain our assertion.

S2. Discussion II: Projected spaces. In order to provide a heuristic understanding of the importance of the projection spaces in feature selection, we give an alternative definition of lower dimensional versions of the input space. For a given input space $\mathcal{X}$, we can define the deleted space $\mathcal{X}^{-J}$ as the space spanned in $\mathbb{R}^{d-|J|}$, obtained by considering only the relevant $d-|J|$ co-ordinates of the vectors from $\mathcal{X}$. This is essentially different from the 
projected space $\mathcal{X}^{J}$, which amounted to looking at the span of the $d-|J|$ co-ordinates only when the remaining $|J|$ co-ordinates are 0 .

The original RFE procedure developed in Guyon et al. [2002] starts off with a given input space $\mathcal{X}$ and eliminates features by re-training the KM algorithm on the lower dimensional spaces $\mathcal{X}^{-J}$. From their discussion, it is seen that if the Gram matrix of the training vectors $\left\{x_{1}, \ldots, x_{n}\right\}$ is given by $\left\{k\left(x_{k}, x_{j}\right)\right\}_{k, j=1}^{n}$, then the Gram matrix of the training vectors $\left\{x_{1}^{-i}, \ldots, x_{n}^{-i}\right\}$ after deleting a particular variable say $\mathcal{X}_{i}$ is taken to be $\left\{k\left(x_{k}^{-i}, x_{j}^{-i}\right)\right\}_{k, j=1}^{n}$. This clearly takes into account the assumption that the kernel $k$ can be defined on deleted vectors as well. It is not intuitively clear however if this can be done for any general kernel $k$. For example, if we start with $\mathcal{X} \subseteq \mathbb{R}^{2}$ and decide to look at only the first feature, then we are restricting ourselves to looking at vectors only from $\mathbb{R}$. Suppose now that the original kernel $k$ was defined on $\mathbb{R}^{2} \times \mathbb{R}^{2}$ as $k(x, y)=x^{\prime} A y$ for a given $2 \times 2$ matrix $\mathrm{A}$. It is not clear then how it can be defined on $\mathbb{R} \times \mathbb{R}$ without breaking its form.

However, for a radial or dot-product kernel, this connection can indeed be achieved. It can be shown that these special kernels have the ability to be redefined on deleted spaces quite easily, and in these cases, they become the same as their projected versions which we propose to use. But for any general kernel, there might not be any "natural" kernel to use in this regard. Hence, in this work we propose to use the projected space $\mathcal{X}^{J}$ instead of the deleted space $\mathcal{X}^{-J}$, as this approach allows a natural way to redefine the kernels in any general situation.

S3. Discussion III: Additional results. We start off with the following lemma:

Lemma S4. Let $\left(\mathcal{F},\|\cdot\|_{\mathcal{F}}\right)$ be a separable functional space, such that the metric $\|\cdot\|_{\mathcal{F}}$ dominates pointwise convergence. Also we assume sup $\|f\|_{\mathcal{F}} \leq$ $C$ for some $C<\infty$ for all $f \in \mathcal{F}$. Let $L$ be such that $L(x, y, f(x)) \leq B$ for some $B<\infty$ for all $f \in \mathcal{F}$. Also assume that for fixed $n \geq 1, \exists$ constants $a \geq 1$ and $p \in(0,1)$ such that $\mathbb{E}_{D_{\mathcal{X}} \sim P_{\mathcal{X}}^{n}} e_{i}\left(\mathcal{F}, L_{\infty}\left(D_{\mathcal{X}}\right)\right) \leq a i^{-\frac{1}{2 p}}, \quad i \geq 1$. Then, we have with probability greater than or equal to $1-e^{-\tau}$,

$$
\begin{aligned}
& \sup _{f \in \mathcal{F}}\left|\mathcal{R}_{L, P}(f)-\mathcal{R}_{L, D}(f)\right| \leq 2 B \sqrt{\frac{2 \tau}{n}}+\frac{10 B \tau}{3 n} \\
& \quad+4 \max \left\{C_{1}(p) c_{L}(C)^{p} a^{p} B^{1-p} n^{-\frac{1}{2}}, C_{2}(p) c_{L}(C)^{\frac{2 p}{1+p}} a^{\frac{2 p}{1+p}} B^{\frac{1-p}{1+p}} n^{-\frac{1}{1+p}}\right\} .
\end{aligned}
$$

for constants $C_{1}(p), C_{2}(p)$ depending only on $p$. 
See Supplementary Section S9.3 for a proof. This lemma gives us a bound for the difference between the empirical risk of a function $f \in \mathcal{F}$ and its omniscient oracle risk. We now assume the premise of Condition $2 \mathrm{a}^{*}$. The above lemma helps set up the next proposition, which aims to bound the difference in the regularized empirical risk of the empirical KM solutions obtained from spaces lying in the pathway hypothesized in Assumption (A1) in Section 3.3.

Proposition S5. Again we assume $P$ to be a probability measure on $\mathcal{X} \times \mathcal{Y}$, and that the input space $\mathcal{X}$ is a valid metric space. We will assume $L$ satisfies $L(x, y, 0) \leq 1$ for all $(x, y) \in \mathcal{X} \times \mathcal{Y}$. Again consider $H$ with kernel $k$ such that $\|k\|_{\infty} \leq 1$, and that for fixed $n \geq 1, \exists$ constants $a \geq 1$ and $p \in(0,1)$ such that $\mathbb{E}_{D_{\mathcal{X}} \sim P_{\mathcal{X}}^{n}} e_{i}\left(i d: H \mapsto L_{\infty}\left(D_{\mathcal{X}}\right)\right) \leq a i^{-\frac{1}{2 p}}, i \geq 1$. Now for a fixed $\lambda>0, \epsilon>0, \tau>0$, and $n \geq 1$, and for $J_{1}, J_{2} \in \tilde{\mathcal{J}}$ such that $J_{1} \subseteq J_{2} \subseteq J_{*}$, we have with probability $P^{n}$ not less than $1-2 e^{-\tau}$,

$$
\begin{aligned}
& \left|\mathcal{R}_{L, D, H^{J_{2}}}^{r e g, \lambda}\left(f_{D, \lambda, H^{J_{2}}}\right)-\mathcal{R}_{L, D, H^{J_{1}}}^{\text {reg, } \lambda}\left(f_{D, \lambda, H^{J_{1}}}\right)\right| \\
& <A_{2}{ }^{J_{1}}(\lambda)+A_{2}^{J_{2}}(\lambda)+12 B \sqrt{\frac{2 \tau}{n}}+20 B \frac{\tau}{n}+24 K_{2} B^{1-p}\left(\frac{a^{2 p}}{\lambda^{p} n}\right)^{\frac{1}{2}},
\end{aligned}
$$

where $A_{2}{ }^{J_{1}}(\lambda)$ and $A_{2}{ }^{J_{2}}(\lambda)$ are the approximation errors for the two separate $R K H S$ classes $H^{J_{1}}$ and $H^{J_{2}}, B:=c_{L}\left(\lambda^{-1 / 2}\right) \lambda^{-1 / 2}+1$, and $K_{2}:=$ $\max \left\{B^{p} / 4, C_{1}(p) c_{L}\left(\lambda^{-\frac{1}{2}}\right)^{p}, C_{2}(p) c_{L}\left(\lambda^{-\frac{1}{2}}\right)^{\frac{2 p}{1+p}}\right\}$ is a constant depending only on $B, p$ and the Lipschitz constant $c_{L}\left(\lambda^{-1 / 2}\right)$.

See Supplementary Section S9.4 for a detailed proof of Proposition S5.

Note that since $B \geq 1$ and $K_{2} \geq B^{p} / 4$, we have that if $a^{2 p}>\lambda^{p} n$,

$$
\begin{aligned}
& \left|\mathcal{R}_{L, D, H^{J}}^{\mathrm{reg}, \lambda}\left(f_{D, \lambda, H^{J}}\right)-\mathcal{R}^{*}{ }_{L, P, H^{J}}\right| \\
& \leq \mathcal{R}_{L, D}(0)+\mathcal{R}_{L, P}(0) \leq 2<3 B \leq 12 K_{2} B^{1-p}\left(\frac{a^{2 p}}{\lambda^{p} n}\right)^{\frac{1}{2}} .
\end{aligned}
$$

Similarly, since $B \geq 1$ and $K_{2} \geq B^{p} / 4$, we have for $a^{2 p}>\lambda^{p} n$,

$$
\begin{aligned}
& \mathcal{R}_{L, P, H^{J}}^{\mathrm{reg}, \lambda}\left(f_{D, \lambda, H^{J}}\right)-\mathcal{R}_{L, P, H^{J}}^{*} \\
& \leq \lambda\left\|f_{D, \lambda, H^{J}}\right\|_{H^{J}}^{2}+\mathcal{R}_{L, D}\left(f_{D, \lambda, H^{J}}\right)+\mathcal{R}_{L, P}\left(f_{D, \lambda, H^{J}}\right) \\
& \leq \mathcal{R}_{L, P}(0)+\mathcal{R}_{L, P}\left(f_{D, \lambda, H^{J}}\right) \leq 1+B \leq 2 B \leq 8 K_{2} B^{1-p}\left(\frac{a^{2 p}}{\lambda^{p} n}\right)^{\frac{1}{2}} .
\end{aligned}
$$

Consequently we obtain the following two corollaries: 
Corollary S6. Assume the conditions of Proposition S5. For any J and all $\epsilon>0, \tau>0$, and $n \geq 1$, we have with $P^{n}$ probability $>1-e^{-\tau}$,

$$
\begin{aligned}
&\left|\mathcal{R}_{L, D, H^{J}}^{r e g, \lambda}\left(f_{D, \lambda, H^{J}}\right)-\mathcal{R}^{*}{ }_{L, P, H^{J}}\right| \\
&<A_{2}{ }^{J}(\lambda)+6 B \sqrt{\frac{2 \tau}{n}}+10 B \frac{\tau}{n}+12 K_{2} B^{1-p}\left(\frac{a^{2 p}}{\lambda^{p} n}\right)^{\frac{1}{2}},
\end{aligned}
$$

where $K_{2}$ is as before. Additionally, if $J \in \tilde{\mathcal{J}}$, we can replace $\mathcal{R}^{*}{ }_{L, P, \mathcal{F}^{J}}$ in the above inequality by $\mathcal{R}^{*}{ }_{L, P, \mathcal{F}}$.

Corollary S7. Oracle Inequality for KM: Assume the conditions of Proposition S5. For any $J$ and all $\epsilon>0, \tau>0$, and $n \geq 1$, we have with $P^{n}$ probability $>1-e^{-\tau}$,

$$
\begin{aligned}
& \mathcal{R}_{L, P, H^{J}}^{r e g, \lambda}\left(f_{D, \lambda, H^{J}}\right)-\mathcal{R}_{L, P, H^{J}}^{*} \\
& <A_{2}{ }^{J}(\lambda)+4 B \sqrt{\frac{2 \tau}{n}}+\frac{20 B \tau}{3 n}+8 K_{2} B^{1-p}\left(\frac{a^{2 p}}{\lambda^{p} n}\right)^{\frac{1}{2}},
\end{aligned}
$$

where $K_{2}$ is as before.

Proposition S5 and Corollaries S6 and S7 jointly imply Lemma 4 given in Section 4.

S4. Discussion IV: Additional Case Studies. Here in this section, we discuss a couple of additional case studies to further demonstrate the applicability and generality of the risk-RFE algorithm.

S4.1. Case Study 3: Linear regression as a linear KM problem with zero regularization. In a linear regression model, the functional relationship is expressed as $y=\langle\alpha, x\rangle+b_{0}$, where $\langle\alpha, x\rangle$ denotes the Euclidean inner product of vectors $\alpha$ and $x$, and $b_{0}$ is the bias. The prediction quality of this model can be measured by the squared-error loss function $L_{L S}$ given as $L_{L S}(x, y, f(x))=(f(x)-y)^{2}$. Our goal is to find linear weights $\widehat{\alpha}$ and $\widehat{b}_{0}$ for the observed data $D$ that minimize the empirical risk. It can be shown that the the regularity conditions required for consistency of our recursive algorithm continue to hold in this setting for this problem.

The risk-RFE procedure presented in this paper translates in the linear regression case to a non-parametric backward selection method based on the value of the 'average sum of squares of error' or $R^{2}$. Note that under restrictive distributional assumptions on the output vector $\mathcal{Y}$ in a non-parametric 
setup, the idea of using penalized versions of $\log R^{2}$ such as AIC, AICc or BIC are well accepted methodologies for model selection. It must be noted that in linear regression risk-RFE can only be used when $d \leq n$ as identifiability becomes an issue otherwise.

S4.2. Case Study 4: Protein classification with mismatch string kernels. A very fundamental problem in computational biology is the classification of proteins into functional and structural classes based on homology of protein sequence data. A mismatch string kernel is used with the kernel machines algorithm in a discriminative approach to solve the protein classification problem. This kernel measures sequence similarity based on shared occurrences of $k$ length subsequences, counted with up to $m$ mismatches. We are still limited to the typical classification problem with $\mathcal{Y}=\{1,-1\}$ and the hinge loss function $L_{H L}(x, y, f(x))=\max \{0,1-y f(x)\}$ as the surrogate. The $(k, m)$ mismatch kernel [see Leslie et al., 2004, for details] is based on a feature map from the space of all finite sequences from an alphabet $\mathcal{A}$ (with $\mathcal{C}(A)=l)$ to $\mathbb{Z}_{\geq 0} l^{k}$, where $l^{k}$ denotes the dimensions spanned by the set of $k$-length subsequences (' $k$-mers') from $\mathcal{A}$. For a fixed $k$-mer $\alpha=a_{1} a_{2} \ldots a_{k}$, with each $a_{i}$ a character in $\mathcal{A}$, the $(k, m)$-neighborhood generated by $\alpha$ is the set of all $k$-length sequences $\beta$ from $\mathcal{A}$ that differ from $\alpha$ by at most $m$ mismatches. We call this set $N_{(k, m)}(\alpha)$.

The feature map $\Phi_{(k, m)}$ for a $k$-mer $\alpha$ is defined as $\Phi_{(k, m)}(\alpha)=$ $\left(\phi_{\beta}(\alpha)\right)_{\beta \in \mathcal{A}^{k}}$, where $\phi_{\beta}(\cdot)$ is a indicator function such that, $\phi_{\beta}(\alpha)=1$ if $\beta \in N_{(k, m)}(\alpha)$, and 0 otherwise. Then for a sequence $x$ of any length, the feature map $\Phi_{k, m}$ is defined as follows:

$$
\Phi_{(k, m)}(x)=\sum_{k-\text { mers } \alpha \text { in } x} \Phi_{(k, m)}(\alpha),
$$

that is, we extend the feature map additively by summing the feature vectors for all the $k$-mers in $x$. The $(k, m)$-mismatch kernel $K_{(k, m)}(x, y)$ is then the Euclidean inner product in the space of feature vectors:

$$
K_{(k, m)}(x, y)=\left\langle\Phi_{(k, m)}(x), \Phi_{(k, m)}(y)\right\rangle .
$$

For $m=0$, we retrieve the $k$-spectral kernel. The kernel can be further normalized as

$$
K_{(k, m)}^{\mathrm{norm}}(x, y)=\frac{K_{(k, m)}(x, y)}{\sqrt{K_{(k, m)}(x, x)} \sqrt{K_{(k, m)}(x, y)}} .
$$

Feature selection in the context of protein classification is conducted on the $k$-mers obtained from a protein sequence instead of the original one [see 
Leslie et al., 2004; Iqbal et al., 2014]. It can be observed that the RKHS produced by the string kernel is finite dimensional and hence satisfies the regularity conditions on the RKHS trivially, and hence, the coordinates of the transformed space (the $k$-mers) can be used directly for feature selection. The problem reduces down to feature selection in linear KMs (produced by the Euclidean inner product), and the applicability of recursive feature selection becomes clear in the context of the discussions we had on the case studies in Supplementary Section S4.1 and proof of Lemma 5 in Supplementary Section S8.3.

S5. Simulations I: Performance of risk-RFE in the nonlinear space. In this section, we look at some simple non-linear settings in both classification and regression to ascertain the performance of risk-RFE when the underlying functional form of the decision function is non-linear. For classification, we consider the hinge loss $L_{H L}$ as the surrogate replacement of the $0-1$ loss, and the KM function is computed using the Gaussian RBF kernel $k_{\gamma}\left(x_{1}, x_{2}\right)=\exp \left\{-\frac{1}{\gamma^{2}}\left\|x_{1}-x_{2}\right\|_{2}^{2}\right\}$, while in the regression setting, we use the Gaussian RBF kernel with the $\epsilon$-insensitive loss $L_{\epsilon}(x, y, f(x))=$ $\max \{0,|y-f(x)|-\epsilon\}$. The value of $\epsilon$ was fixed at 0.1 . In classification, we look at three different scenarios with increasing design size but fixed number of signals, namely (a) $d=10, d_{0}=2$, (b) $d=50, d_{0}=2$, (c) $d=100, d_{0}=2$. While in regression, we look at three different scenarios with both increasing design size and increasing signal size, namely (a) $d=10, d_{0}=2$, (b) $d=50$, $d_{0}=3,(\mathrm{c}) d=100, d_{0}=4$.

We again consider two settings: (i) absence of colinearity or (ii) presence of colinearity in covariates. In all of the above settings, we generate covariates uniformly from the Gaussian distribution $X \in \mathbb{R} \sim N_{d}(0, \Sigma)$, where $\Sigma$ is diagonal in the case when there is no colinearity, but set some of the offdiagonal terms to be nonzero to induce correlation between the signals when colinearity is present. In classification, $Y$ takes two labels: 1 inside the square formed by $\left(-a \leq X_{1} \leq a, \ldots,-a \leq X_{2} \leq a\right)$, and -1 outside this square. The constant $a$ is chosen such that probability of $Y=1$ is the same as that of $Y=-1$. In regression, $Y$ is generated as $Y=\frac{a_{1} X_{1} X_{2}}{\left(1+b_{1} X_{1}\right)^{2}}+\cdots+$ $\frac{a_{d_{0}-1} X_{d_{0}-1} X_{d_{0}}}{\left(1+b_{d_{0}-1} X_{d_{0}-1}\right)^{2}}+\frac{a_{d_{0}} X_{d_{0}} X_{1}}{\left(1+b_{d_{0}} X_{d_{0}}\right)^{2}}$, where $a_{i}, b_{i}$ for $i=1, \ldots, d_{0}$ are some constants.

We initialize the original KM function using a 5-fold cross validation on the kernel width $\gamma$ and the regularization parameter $\lambda$ and they are chosen from the set of values $\left(\frac{2}{n \lambda}, \gamma\right)=\left(0.01 \times 10^{i}, j\right), i=\{0,1,2,3,4,5\}, j=$ $\{1,2,3,4,5\}$, where $n$ is the sample size for the given setting. The procedures are repeated for different sample sizes $n=\{100,200,400,800\}$, and repeated 100 times. The algorithms are implemented in MATLAB. For implementa- 


\begin{tabular}{|c|c|c|c|c|c|c|c|c|c|c|c|c|c|c|c|c|}
\hline \multirow{2}{*}{$\begin{array}{l}\text { Classification } \\
d=10, d_{0}=2\end{array}$} & \multicolumn{4}{|c|}{$n=100$} & \multicolumn{4}{|c|}{$n=200$} & \multicolumn{4}{|c|}{$n=400$} & \multicolumn{4}{|c|}{$n=800$} \\
\hline & $\begin{array}{c}\% \\
\text { no } \\
\text { error }\end{array}$ & $\begin{array}{c}\% \\
1 \\
\text { error }\end{array}$ & $\begin{array}{l}\% \\
>1 \\
\text { error }\end{array}$ & $\begin{array}{c}\text { Misclass. } \\
\text { (Standard } \\
\text { Error) }\end{array}$ & $\begin{array}{c}\% \\
\text { no } \\
\text { error }\end{array}$ & $\begin{array}{c}\% \\
1 \\
\text { error }\end{array}$ & $\begin{array}{c}\% \\
>1 \\
\text { error }\end{array}$ & $\begin{array}{c}\text { Misclass. } \\
\text { (Standard } \\
\text { Error) }\end{array}$ & \begin{tabular}{|c}
$\%$ \\
no \\
error
\end{tabular} & $\begin{array}{c}\% \\
1 \\
\text { error }\end{array}$ & $\begin{array}{c}\% \\
>1 \\
\text { error }\end{array}$ & $\begin{array}{c}\text { Misclass. } \\
\text { (Standard } \\
\text { Error) }\end{array}$ & $\begin{array}{c}\% \\
\text { no } \\
\text { error }\end{array}$ & $\begin{array}{c}\% \\
1 \\
\text { error }\end{array}$ & $\begin{array}{c}\% \\
>1 \\
\text { error }\end{array}$ & $\begin{array}{l}\text { Misclass. } \\
\text { (Standard } \\
\text { Error) }\end{array}$ \\
\hline KM-G GRFE (NR) & 96 & 0 & 4 & $0.13(0.05)$ & 98 & 2 & 0 & $0.10(0.02)$ & 100 & 0 & 0 & $0.10(0.02)$ & 100 & 0 & 0 & $0.06(0.01)$ \\
\hline KM-G RRFE & 100 & 0 & 0 & $0.14(0.06)$ & 100 & 0 & 0 & $0.11(0.03)$ & 100 & 0 & 0 & $0.10(0.02)$ & 100 & 0 & 0 & $0.06(0.01)$ \\
\hline SCAD SVM & 1 & 43 & 56 & $0.40(0.14)$ & 2 & 27 & 71 & $0.42(0.13)$ & 0 & 36 & 64 & $0.42(0.11)$ & 0 & 28 & 72 & $0.43(0.13)$ \\
\hline Log Reg Lasso & 0 & 14 & 86 & $0.50(0.05)$ & 5 & 27 & 68 & $0.49(0.04)$ & 2 & 28 & 70 & $0.49(0.03)$ & 4 & 20 & 76 & $0.50(0.02)$ \\
\hline KM-G RRFE (NR) & 65 & 21 & 14 & $0.33(0.17)$ & 86 & 14 & 0 & $0.21(0.15)$ & 100 & 0 & 0 & $0.10(0.07)$ & 100 & 0 & 0 & $0.08(0.01)$ \\
\hline $\mathrm{KM}$ & 61 & 22 & 17 & $0.35(0.16)$ & 83 & 10 & 7 & $0.24(0.17)$ & 98 & 0 & 2 & $0.11(0.08)$ & 100 & 0 & 0 & 0.08 \\
\hline $\mathrm{KM}$ & 72 & 22 & 6 & $0.39(0.16)$ & 88 & 12 & 0 & $0.29(0.07)$ & 100 & 0 & 0 & $0.18(0.09)$ & 100 & 0 & 0 & 0.08 \\
\hline $\mathrm{SCA}$ & 30 & 29 & 41 & $0.49(0.05)$ & 0 & 7 & 93 & $0.48(0.05)$ & 0 & 1 & 99 & $0.49(0.04)$ & 0 & 4 & 96 & $0.49(0.06)$ \\
\hline KM-G RRFE (NR) & 55 & 15 & 30 & 1 & 68 & 6 & 26 & ) & 87 & 3 & 10 & 0.1 & 94 & 2 & 4 & 2) \\
\hline $\mathrm{KM}$ & 33 & 18 & 49 & $0.41(\mathrm{C}$ & 44 & 22 & 34 & $0.32(\mathrm{C}$ & 72 & 1 & 27 & $0.24(\mathrm{C}$ & 78 & 0 & 22 & 0.20( \\
\hline $\mathrm{KN}$ & 58 & 18 & 24 & 0.40( & 70 & 4 & 26 & 0.36( & 89 & 1 & 10 & $0.28(0.04)$ & 94 & 2 & 4 & 0.24 \\
\hline & 45 & 12 & 43 & 0.4 & 30 & 17 & 53 & 0.48 & 0 & 2 & 98 & 0.49( & 0 & 0 & 100 & 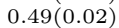 \\
\hline Lo & 0 & 3 & 97 & $0.50(0.05)$ & 0 & 2 & 98 & 0.49( & 0 & 1 & 99 & $0.50(0$ & 0 & 6 & 94 & 0.48( \\
\hline & NA & NA & NA & $0.49(0.06)$ & NA & NA & NA & $0.49(\mathrm{C}$ & NA & NA & NA & $0.47(0$ & NA & NA & NA & $0.47(0.02)$ \\
\hline $\begin{array}{l}\text { Classification } \\
\text { (with colinearity) }\end{array}$ & & & $=10$ & & & & 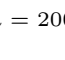 & & & & 0 & & & & 30 & \\
\hline$d=$ & $\begin{array}{c}\% \\
\text { no } \\
\text { error }\end{array}$ & $\begin{array}{c}\% \\
1 \\
\text { erro }\end{array}$ & $\begin{array}{c}\% \\
>1 \\
\text { error }\end{array}$ & $\begin{array}{c}\text { Misclass. } \\
\text { (Standard } \\
\text { Error) }\end{array}$ & $\begin{array}{c}\% \\
\text { no } \\
\text { error }\end{array}$ & $\begin{array}{c}\% \\
1\end{array}$ & $\begin{array}{c}\% \\
>1 \\
\text { error }\end{array}$ & $\begin{array}{c}\text { Misclass. } \\
\text { (Standard } \\
\text { Error) }\end{array}$ & \begin{tabular}{|c|}
$\%$ \\
no \\
error
\end{tabular} & $\begin{array}{c}\% \\
1 \\
\text { error }\end{array}$ & $\begin{array}{c}\% \\
>1 \\
\text { error }\end{array}$ & $\begin{array}{c}\text { Misclass. } \\
\text { (Standard } \\
\text { Error) }\end{array}$ & $\begin{array}{c}\% \\
\text { no } \\
\text { error }\end{array}$ & $\begin{array}{l}\% \\
1 \\
\text { rror }\end{array}$ & $\begin{array}{c}\% \\
>1 \\
\text { error }\end{array}$ & $\begin{array}{l}\text { Misclass. } \\
\text { (Standard } \\
\text { Error) }\end{array}$ \\
\hline B) & 100 & 0 & 0 & .13 & 100 & 0 & 0 & 0.1 & 100 & 0 & 0 & 0.0 & 100 & 0 & 0 & 0.0 \\
\hline K & 69 & 20 & 11 & 0.32 & 94 & 3 & 3 & & 100 & 0 & 0 & & 100 & 0 & 0 & 0.0 \\
\hline & 61 & 22 & 17 & 0.3 & 88 & 6 & 6 & 0.22 & 90 & 0 & 10 & 0.16 & 99 & 1 & 0 & \\
\hline & 75 & 18 & 7 & & 95 & 2 & 3 & 0.28 & 100 & 0 & 0 & $0.21(0.08)$ & 100 & 0 & 0 & \\
\hline & 15 & 50 & 35 & $0.49(0.06)$ & 3 & 7 & 90 & $0.48(0.0$ & 0 & 1 & 99 & $0.50(0.04)$ & 0 & 26 & 74 & $0.42(0.12)$ \\
\hline & 0 & 3 & 97 & $0.51(0.05)$ & 0 & 2 & 98 & $0.50(0$ & 1 & 1 & 98 & $0.50(0.03)$ & 0 & 10 & 90 & $0.50(0$ \\
\hline & NA & NA & NA & 0.49 ( & NA & NA & NA & 0.46 & NA & NA & NA & 0.44( & NA & NA & NA & 0.3 \\
\hline$d=$ & $\begin{array}{c}\% \\
\text { no } \\
\text { errol }\end{array}$ & $\begin{array}{c}\% \\
1 \\
\text { erro }\end{array}$ & $\begin{array}{c}\% \\
>1 \\
\text { error }\end{array}$ & $\begin{array}{c}\text { Misclass. } \\
\text { (Standard } \\
\text { Error) }\end{array}$ & $\begin{array}{c}\% \\
\text { no } \\
\text { error }\end{array}$ & $\begin{array}{c}\% \\
1 \\
\text { erro }\end{array}$ & $\begin{array}{c}\% \\
>1 \\
\text { error }\end{array}$ & $\begin{array}{c}\text { Misclass. } \\
\text { (Standard } \\
\text { Error) }\end{array}$ & \begin{tabular}{|c|}
$\%$ \\
no \\
error
\end{tabular} & $\begin{array}{c}\% \\
1 \\
\text { erro }\end{array}$ & $\begin{array}{c}\% \\
>1 \\
\text { error }\end{array}$ & $\begin{array}{c}\text { Misclass. } \\
\text { (Standard } \\
\text { Error) }\end{array}$ & $\begin{array}{c}\% \\
\text { no } \\
\text { error }\end{array}$ & $\begin{array}{c}\% \\
1 \\
\text { error }\end{array}$ & $\begin{array}{c}\% \\
>1 \\
\text { error }\end{array}$ & $\begin{array}{l}\text { Misclass. } \\
\text { (Standard } \\
\text { Error) }\end{array}$ \\
\hline & 5. & 11 & 36 & & 5 & 5 & 28 & & 86 & 0 & 14 & 0. & 96 & 0 & 4 & \\
\hline WE (NK) & 34 & 10 & 56 & $0.43(0.13)$ & 47 & 15 & 38 & $0.36(0.17)$ & 62 & 9 & 29 & $0.26(0.18)$ & 76 & 10 & 14 & $0.19(0.14)$ \\
\hline & 55 & 12 & 33 & $0.41(0$ & 72 & 3 & 25 & $0.38(0$. & 86 & 0 & 14 & $0.29(0.09)$ & 96 & 0 & 4 & $0.23(0.06)$ \\
\hline & 42 & 20 & 38 & & 27 & 9 & 64 & & 0 & 2 & 98 & & 0 & 0 & 100 & \\
\hline & 0 & 7 & 93 & $0.49(\mathrm{C}$ & 0 & 2 & 98 & $0.50(0$ & 0 & 2 & 98 & $0.49(0.03)$ & 0 & 2 & 98 & $0.50(0.02)$ \\
\hline No Selection & NA & NA & NA & $0.50(0.05)$ & NA & NA & NA & $0.50(0.04)$ & NA & NA & NA & $0.47(0.03)$ & NA & NA & NA & $0.46(0.02)$ \\
\hline
\end{tabular}

TABLE S5.1. Comparison in classification between (a) KM-G RRFE (NR)- risk-RFE with KM Gauss with naive ranks, (b) KM-G GRFE (NR)- Guyon RFE with KM Gauss with naive ranks, (c) KM-G RRFE (CP)- risk-RFE with KM Gauss with change point model, (d) SCAD SVM- linear KM (SVM) with SCAD, (e) Log Reg Lasso- logistic regression with LASSO, and ( $f$ ) No Selection-KM Gauss without selection under nonlinearity, without and with the presence of colinearity.

tion of the KM functions, we use the SPIDER library for MATLAB ${ }^{1}$. It

\footnotetext{
${ }^{1}$ The Spider library can be found at http://www.kyb.tuebingen.mpg.de/bs/people/
} 
already has a feature elimination algorithm based on Guyon's RFE, and we modified it here to suit our criterion for deletion.

As a first confirmatory step, we decide to compare the performance of risk-RFE with that of Guyon's RFE to see if we indeed have enhanced performance in consistency of feature selection. Because the number of important features is known in each setting, we also formulate a conservative approach called naive ranks (NR), to make it comparable to feature selection using Guyon's RFE, as the latter can not choose a subset on its own. Here, we only consider the first $d_{0}$ number of highest ranked signals, and make predictions based on reduced models containing only these features. We also compare it with linear selection methods such as SCAD-SVM (linear KM with SCAD) and logistic regression with LASSO in classification, and with linear regression with LASSO in regression, to show the necessity of considering correct feature selection techniques when we cannot assume in advance that the underlying space is linear. As all these methods can choose a subset of features on their own, they can be comparable to our algorithm with the change point $(\mathrm{CP})$ formulation. For penalized methods, we use cross validation to select the amount of penalization that is required in each case. We look at the percentage of times the algorithms (a) make no errors, (b) make only one error, or (c) make multiple errors, and also note the average test prediction error, and their standard errors. In the case of NR formulations, a mistake is made if the rank of any signal is lower than the total number of important features in the model, while in CR, a mistake is made if the chosen set of features does not contain a signal.

Table S5.1 compiles results from the different simulation settings in classification, while table S5.2 compiles those from regression, both under the presence and absence of colinearity. As we can see from both tables, the riskRFE procedures (both RRFE-NR with naive ranks, and RRFE-CP with the change point model) dominate performance in choosing the correct features consistently. This performance is better in lower dimensional settings for a fixed sample size, and for higher sample sizes for a given setting. This supports our asymptotic claims for consistency of the algorithm. In classification, we see that RRFE-CP can sometimes do better than RRFE-NR in terms of feature selection, as the change point model consistently selects more features than the naive rank approach, often, therefore, it can be better at retaining the signals. In regression, however, we do not see this difference.

In comparing GRFE-NR (Guyon's RFE with naive ranks) with RRFE (both CP and NR) w.r.t. feature selection, we see that GRFE does relatively well in classification, although never better than RRFE, and the

spider/ 


\begin{tabular}{|c|c|c|c|c|c|c|c|c|c|c|c|c|c|c|c|c|}
\hline \multirow{3}{*}{\begin{tabular}{|l|} 
Regression \\
$d=10, d_{0}=2$ \\
KM-G RRFE (NR) \\
KM-G GRFE (NR) \\
KM-G RRFE (CP) \\
Lin Reg Lasso \\
No Selection \\
\end{tabular}} & \multicolumn{4}{|c|}{$n=100$} & \multicolumn{4}{|c|}{$n=200$} & \multicolumn{4}{|c|}{$n=400$} & \multicolumn{4}{|c|}{$n=800$} \\
\hline & \begin{tabular}{|c}
$\%$ \\
no \\
error
\end{tabular} & $\begin{array}{c}\% \\
1 \\
\text { error }\end{array}$ & $\begin{array}{c}\% \\
>1 \\
\text { error }\end{array}$ & $\begin{array}{c}\text { Test Err. } \\
\text { (Standard } \\
\text { Error) }\end{array}$ & $\begin{array}{c}\% \\
\text { no } \\
\text { error }\end{array}$ & $\begin{array}{c}\% \\
1 \\
\text { error }\end{array}$ & $\begin{array}{c}\% \\
>1 \\
\text { error }\end{array}$ & $\begin{array}{l}\text { Test Err. } \\
\text { (Standard } \\
\text { Error) }\end{array}$ & $\begin{array}{c}\% \\
\text { no } \\
\text { error }\end{array}$ & $\begin{array}{c}\% \\
1 \\
\text { error }\end{array}$ & $\begin{array}{l}\% \\
>1 \\
\text { error }\end{array}$ & $\begin{array}{l}\text { Test Err. } \\
\text { (Standard } \\
\text { Error) }\end{array}$ & $\begin{array}{c}\% \\
\text { no } \\
\text { error }\end{array}$ & $\begin{array}{c}\% \\
1 \\
\text { error }\end{array}$ & $\begin{array}{c}\% \\
>1 \\
\text { error }\end{array}$ & $\begin{array}{l}\text { Test Err. } \\
\text { (Standard } \\
\text { Error) }\end{array}$ \\
\hline & $\begin{array}{c}100 \\
89 \\
100 \\
2 \\
\text { NA }\end{array}$ & $\begin{array}{c}0 \\
11 \\
0 \\
17 \\
\text { NA }\end{array}$ & $\begin{array}{c}0 \\
0 \\
0 \\
81 \\
\text { NA }\end{array}$ & $\begin{array}{c}0.78(0.35) \\
1.07(0.86) \\
0.89(0.33) \\
2.4(0.41) \\
1.04(0.33) \\
\end{array}$ & $\begin{array}{c}100 \\
100 \\
100 \\
4 \\
\text { NA }\end{array}$ & $\begin{array}{c}0 \\
0 \\
0 \\
14 \\
\text { NA }\end{array}$ & $\begin{array}{c}0 \\
0 \\
0 \\
82 \\
\text { NA }\end{array}$ & $\begin{array}{l}0.48(0.23) \\
0.48(0.23) \\
0.54(0.22) \\
2.31(0.28) \\
0.83(0.22)\end{array}$ & $\begin{array}{c}100 \\
100 \\
100 \\
1 \\
\text { NA }\end{array}$ & $\begin{array}{c}0 \\
0 \\
0 \\
29 \\
\text { NA }\end{array}$ & $\begin{array}{c}0 \\
0 \\
0 \\
70 \\
\text { NA } \\
\end{array}$ & $\begin{array}{l}0.46(0.17) \\
0.46(0.17) \\
0.50(0.17) \\
2.36(0.23) \\
0.75(0.16)\end{array}$ & $\begin{array}{c}100 \\
100 \\
100 \\
2 \\
\text { NA }\end{array}$ & $\begin{array}{c}0 \\
0 \\
0 \\
28 \\
\text { NA }\end{array}$ & $\begin{array}{c}0 \\
0 \\
0 \\
70 \\
\text { NA }\end{array}$ & $\begin{array}{l}0.37(0.10) \\
0.37(0.10) \\
0.39(0.10) \\
2.31(0.15) \\
0.60(0.08)\end{array}$ \\
\hline$d=50, d_{0}=3$ & $\begin{array}{c}\% \\
\text { no } \\
\text { error }\end{array}$ & $\begin{array}{c}\% \\
1 \\
\text { error }\end{array}$ & $\begin{array}{c}\% \\
>1 \\
\text { error }\end{array}$ & $\begin{array}{l}\text { Test Err. } \\
\text { (Standard } \\
\text { Error) }\end{array}$ & $\begin{array}{c}\% \\
\text { no } \\
\text { error }\end{array}$ & $\begin{array}{c}\% \\
1 \\
\text { error }\end{array}$ & $\begin{array}{c}\% \\
>1 \\
\text { error }\end{array}$ & $\begin{array}{l}\text { Test Err. } \\
\text { (Standard } \\
\text { Error) }\end{array}$ & $\begin{array}{c}\% \\
\text { no } \\
\text { error }\end{array}$ & $\begin{array}{c}\% \\
1 \\
\text { error }\end{array}$ & $\begin{array}{c}\% \\
>1 \\
\text { error }\end{array}$ & $\begin{array}{l}\text { Test Err. } \\
\text { (Standard } \\
\text { Error) }\end{array}$ & $\begin{array}{c}\% \\
\text { no } \\
\text { error }\end{array}$ & $\begin{array}{c}\% \\
1 \\
\text { error }\end{array}$ & $\begin{array}{c}\% \\
>1 \\
\text { error }\end{array}$ & $\begin{array}{c}\text { Test Err. } \\
\text { (Standard } \\
\text { Error) }\end{array}$ \\
\hline $\begin{array}{l}\text { KM-G F } \\
\text { KM-G C } \\
\text { KM-G F } \\
\text { Lin Reg } \\
\text { No Selec }\end{array}$ & $\begin{array}{c}100 \\
1 \\
100 \\
0 \\
\text { NA }\end{array}$ & $\begin{array}{c}0 \\
39 \\
0 \\
0 \\
\text { NA }\end{array}$ & $\begin{array}{c}0 \\
60 \\
0 \\
100 \\
\text { NA }\end{array}$ & $\begin{array}{l}4.58(1.17) \\
8.24(1.47) \\
4.68(1.08) \\
7.49(1.19) \\
5.34(0.81)\end{array}$ & $\begin{array}{c}100 \\
8 \\
100 \\
0 \\
\text { NA }\end{array}$ & $\begin{array}{c}0 \\
81 \\
0 \\
3 \\
\text { NA }\end{array}$ & $\begin{array}{c}0 \\
11 \\
0 \\
97 \\
\text { NA }\end{array}$ & $\begin{array}{l}3.05(0.62) \\
5.54(1.57) \\
3.67(0.74) \\
7.90(0.73) \\
4.40(0.62)\end{array}$ & $\begin{array}{c}100 \\
14 \\
100 \\
0 \\
\text { NA }\end{array}$ & $\begin{array}{c}0 \\
82 \\
0 \\
4 \\
\text { NA }\end{array}$ & $\begin{array}{c}0 \\
4 \\
0 \\
96 \\
\text { NA }\end{array}$ & $\begin{array}{l}2.59(0.30) \\
6.03(1.57) \\
3.75(0.49) \\
7.06(0.55) \\
4.09(0.25)\end{array}$ & $\begin{array}{c}100 \\
26 \\
100 \\
2 \\
\text { NA }\end{array}$ & $\begin{array}{c}0 \\
74 \\
0 \\
0 \\
\text { NA }\end{array}$ & $\begin{array}{c}0 \\
0 \\
0 \\
98 \\
\text { NA }\end{array}$ & $\begin{array}{l}2.05(0.15) \\
5.12(1.89) \\
2.82(0.39) \\
6.96(0.26) \\
3.59(0.19)\end{array}$ \\
\hline$d=$ & \begin{tabular}{|c}
$\%$ \\
no \\
error
\end{tabular} & $\begin{array}{c}\% \\
1 \\
\text { error }\end{array}$ & $\begin{array}{l}\% \\
>1 \\
\text { error }\end{array}$ & $\begin{array}{l}\text { Test Err. } \\
\text { (Standard } \\
\text { Error) }\end{array}$ & \begin{tabular}{|c}
$\%$ \\
no \\
error
\end{tabular} & $\begin{array}{c}\% \\
1 \\
\text { error }\end{array}$ & $\begin{array}{c}\% \\
>1 \\
\text { error }\end{array}$ & $\begin{array}{l}\text { Test Err. } \\
\text { (Standard } \\
\text { Error) }\end{array}$ & \begin{tabular}{|c|}
$\%$ \\
no \\
error
\end{tabular} & $\begin{array}{c}\% \\
1 \\
\text { error }\end{array}$ & $\begin{array}{c}\% \\
>1 \\
\text { error }\end{array}$ & $\begin{array}{l}\text { Test Err. } \\
\text { (Standard } \\
\text { Error) }\end{array}$ & $\begin{array}{c}\% \\
\text { no } \\
\text { error }\end{array}$ & $\begin{array}{c}\% \\
1 \\
\text { error }\end{array}$ & $\begin{array}{c}\% \\
>1 \\
\text { error }\end{array}$ & $\begin{array}{l}\text { Test Err. } \\
\text { (Standard } \\
\text { Error) }\end{array}$ \\
\hline $\begin{array}{l}\mathrm{FE}(\mathrm{NR}) \\
\mathrm{FE}(\mathrm{NR}) \\
\mathrm{FE}(\mathrm{CP}) \\
\text { isso } \\
\mathrm{n}\end{array}$ & $\begin{array}{c}8 \\
0 \\
8 \\
0 \\
\text { NA } \\
\end{array}$ & $\begin{array}{c}30 \\
0 \\
30 \\
0 \\
\text { NA }\end{array}$ & $\begin{array}{c}62 \\
100 \\
62 \\
100 \\
\text { NA }\end{array}$ & $\begin{array}{c}9.91(1.96) \\
10.46(1.81) \\
9.84(1.78) \\
10.37(1.76) \\
9.78(1.09) \\
\end{array}$ & $\begin{array}{c}31 \\
0 \\
31 \\
0 \\
\text { NA } \\
\end{array}$ & $\begin{array}{c}58 \\
0 \\
58 \\
1 \\
\text { NA } \\
\end{array}$ & $\begin{array}{c}11 \\
100 \\
11 \\
99 \\
\text { NA }\end{array}$ & $\begin{array}{c}8.95(2.12) \\
10.67(1.60) \\
8.84(1.37) \\
9.52(1.26) \\
8.96(1.19) \\
\end{array}$ & $\begin{array}{c}73 \\
0 \\
73 \\
0 \\
\text { NA } \\
\end{array}$ & $\begin{array}{c}22 \\
0 \\
22 \\
3 \\
\text { NA } \\
\end{array}$ & $\begin{array}{c}5 \\
100 \\
5 \\
97 \\
\text { NA } \\
\end{array}$ & $\begin{array}{l}6.47(1.93) \\
9.81(0.92) \\
6.74(2.02) \\
9.42(0.77) \\
8.57(1.04) \\
\end{array}$ & \begin{tabular}{|c}
98 \\
0 \\
98 \\
0 \\
NA \\
\end{tabular} & $\begin{array}{c}2 \\
0 \\
2 \\
0 \\
\text { NA }\end{array}$ & $\begin{array}{c}0 \\
100 \\
0 \\
100 \\
\text { NA } \\
\end{array}$ & $\begin{array}{l}4.03(0.58) \\
9.03(0.68) \\
4.88(0.52) \\
9.24(0.42) \\
8.27(0.37) \\
\end{array}$ \\
\hline $\begin{array}{l}\text { Regression } \\
\text { (with colinearity) }\end{array}$ & & & $\imath=$ & & & & $n=$ & & & & $=40$ & & & & $=800$ & \\
\hline & \begin{tabular}{|c|}
$\%$ \\
no \\
error
\end{tabular} & $\begin{array}{c}\% \\
1 \\
\text { erro } \\
\end{array}$ & $\begin{array}{c}\% \\
>1 \\
\text { error }\end{array}$ & $\begin{array}{c}\text { (Standard } \\
\text { Error) }\end{array}$ & \begin{tabular}{|c|}
$\%$ \\
no \\
error
\end{tabular} & $\begin{array}{c}\% \\
1 \\
\text { error } \\
\end{array}$ & $\begin{array}{c}\% \\
>1 \\
\text { error }\end{array}$ & $\begin{array}{c}\text { Test Err. } \\
\text { (Standard } \\
\text { Error) }\end{array}$ & $\begin{array}{l}\% \\
\text { no } \\
\text { error }\end{array}$ & $\begin{array}{c}\% \\
1 \\
\text { error } \\
\end{array}$ & $\begin{array}{l}\% \\
>1 \\
\text { error }\end{array}$ & $\begin{array}{c}\text { Test Err. } \\
\text { (Standard } \\
\text { Error) }\end{array}$ & \begin{tabular}{|c|}
$\%$ \\
no \\
error
\end{tabular} & $\begin{array}{c}\% \\
1 \\
\text { error } \\
\end{array}$ & $\begin{array}{c}\% \\
>1 \\
\text { error }\end{array}$ & $\begin{array}{l}\text { Test Err. } \\
\text { (Standard } \\
\text { Error) }\end{array}$ \\
\hline $\begin{array}{l}\text { KM-G RRFE (NR) } \\
\text { KM-G GRFE (NR) } \\
\text { KM-G RRFE (CP) } \\
\text { Lin Reg Lasso } \\
\text { No Selection }\end{array}$ & $\begin{array}{c}100 \\
93 \\
100 \\
0 \\
\text { NA }\end{array}$ & $\begin{array}{c}0 \\
7 \\
0 \\
13 \\
\text { NA }\end{array}$ & $\begin{array}{c}0 \\
0 \\
0 \\
87 \\
\text { NA }\end{array}$ & $\begin{array}{l}0.75(0.41) \\
0.90(0.67) \\
0.90(0.41) \\
2.11(0.34) \\
1.13(0.28)\end{array}$ & $\begin{array}{c}100 \\
100 \\
100 \\
7 \\
\text { NA }\end{array}$ & $\begin{array}{c}0 \\
0 \\
0 \\
18 \\
\text { NA }\end{array}$ & $\begin{array}{c}0 \\
0 \\
0 \\
75 \\
\text { NA }\end{array}$ & $\begin{array}{l}0.56(0.26) \\
0.56(0.26) \\
0.64(0.26) \\
2.19(0.31) \\
0.87(0.24)\end{array}$ & $\begin{array}{c}100 \\
100 \\
100 \\
4 \\
\text { NA }\end{array}$ & $\begin{array}{c}0 \\
0 \\
0 \\
4 \\
\text { NA }\end{array}$ & $\begin{array}{c}0 \\
0 \\
0 \\
92 \\
\text { NA } \\
\end{array}$ & $\begin{array}{l}0.36(0.13) \\
0.36(0.13) \\
0.39(0.13) \\
2.07(0.18) \\
0.65(0.13)\end{array}$ & $\begin{array}{c}100 \\
100 \\
100 \\
0 \\
\text { NA }\end{array}$ & $\begin{array}{c}0 \\
0 \\
0 \\
16 \\
\text { NA }\end{array}$ & $\begin{array}{c}0 \\
0 \\
0 \\
84 \\
\text { NA } \\
\end{array}$ & $\begin{array}{l}0.34(0.10) \\
0.34(0.10) \\
0.36(0.09) \\
2.08(0.12) \\
0.55(0.08)\end{array}$ \\
\hline$d=$ & \begin{tabular}{|c|}
$\%$ \\
no \\
error
\end{tabular} & $\begin{array}{c}\% \\
1 \\
\text { erro } \\
\end{array}$ & $\begin{array}{l}\% \\
>1 \\
\text { error }\end{array}$ & $\begin{array}{c}\text { Test Err. } \\
\text { (Standard } \\
\text { Error) }\end{array}$ & $\begin{array}{c}\% \\
\text { no } \\
\text { error }\end{array}$ & $\begin{array}{c}\% \\
1 \\
\text { erro } \\
\end{array}$ & $\begin{array}{l}\% \\
>1 \\
\text { error }\end{array}$ & $\begin{array}{c}\text { Test Err. } \\
\text { (Standard } \\
\text { Error) }\end{array}$ & \begin{tabular}{|c|}
$\%$ \\
no \\
error
\end{tabular} & $\begin{array}{c}\% \\
1 \\
\text { error } \\
\end{array}$ & $\begin{array}{l}\% \\
>1 \\
\text { error }\end{array}$ & $\begin{array}{c}\text { Test Err. } \\
\text { (Standard } \\
\text { Error) }\end{array}$ & $\begin{array}{c}\% \\
\text { no } \\
\text { error }\end{array}$ & $\begin{array}{c}\% \\
1 \\
\text { error } \\
\end{array}$ & $\begin{array}{c}\% \\
>1 \\
\text { error }\end{array}$ & $\begin{array}{c}\text { Test Err. } \\
\text { (Standard } \\
\text { Error) }\end{array}$ \\
\hline $\begin{array}{l}\text { KM-G RRFE (NR) } \\
\text { KM-G GRFE (NR) } \\
\text { KM-G RRFE (CP) } \\
\text { Lin Reg Lasso } \\
\text { No Selection }\end{array}$ & $\begin{array}{c}91 \\
14 \\
91 \\
0 \\
\text { NA }\end{array}$ & $\begin{array}{c}9 \\
53 \\
9 \\
6 \\
\text { NA } \\
\end{array}$ & $\begin{array}{c}0 \\
33 \\
0 \\
94 \\
\text { NA }\end{array}$ & $\begin{array}{l}4.18(1.09) \\
7.05(2.76) \\
4.50(0.97) \\
8.25(1.55) \\
5.37(0.63) \\
\end{array}$ & $\begin{array}{c}100 \\
7 \\
100 \\
0 \\
\text { NA }\end{array}$ & $\begin{array}{c}0 \\
84 \\
0 \\
4 \\
\text { NA }\end{array}$ & $\begin{array}{c}0 \\
9 \\
0 \\
96 \\
\text { NA }\end{array}$ & $\begin{array}{l}3.14(0.60) \\
5.72(1.48) \\
3.81(0.71) \\
7.99(0.73) \\
4.58(0.62)\end{array}$ & $\begin{array}{c}100 \\
29 \\
100 \\
0 \\
\text { NA }\end{array}$ & $\begin{array}{c}0 \\
69 \\
0 \\
0 \\
\text { NA }\end{array}$ & $\begin{array}{c}0 \\
2 \\
0 \\
100 \\
\text { NA } \\
\end{array}$ & $\begin{array}{l}2.25(0.29) \\
4.38(1.69) \\
2.89(0.56) \\
7.56(0.40) \\
3.71(0.59) \\
\end{array}$ & $\begin{array}{c}100 \\
48 \\
100 \\
0 \\
\text { NA }\end{array}$ & $\begin{array}{c}0 \\
52 \\
0 \\
0 \\
\text { NA }\end{array}$ & $\begin{array}{c}0 \\
0 \\
0 \\
100 \\
\text { NA }\end{array}$ & $\begin{array}{l}1.85(0.18) \\
3.09(1.49) \\
2.16(0.25) \\
7.53(0.22) \\
3.35(0.17) \\
\end{array}$ \\
\hline$d$ & \begin{tabular}{|c|}
$\%$ \\
no \\
error
\end{tabular} & $\begin{array}{c}\% \\
1 \\
\text { erro }\end{array}$ & $\begin{array}{c}\% \\
>1 \\
\text { error }\end{array}$ & $\begin{array}{c}\text { Test Err. } \\
\text { (Standard } \\
\text { Error) }\end{array}$ & $\begin{array}{c}\% \\
\text { no } \\
\text { error }\end{array}$ & $\begin{array}{c}\% \\
1 \\
\text { erro } \\
\end{array}$ & $\begin{array}{c}\% \\
>1 \\
\text { error }\end{array}$ & $\begin{array}{l}\text { Test Err. } \\
\text { (Standard } \\
\text { Error) }\end{array}$ & \begin{tabular}{|c|}
$\%$ \\
no \\
error
\end{tabular} & $\begin{array}{c}\% \\
1 \\
\text { error }\end{array}$ & $\begin{array}{c}\% \\
>1 \\
\text { error }\end{array}$ & $\begin{array}{l}\text { Test Err. } \\
\text { (Standard } \\
\text { Error) }\end{array}$ & \begin{tabular}{|c}
$\%$ \\
no \\
error
\end{tabular} & $\begin{array}{c}\% \\
1 \\
\text { error }\end{array}$ & $\begin{array}{c}\% \\
>1 \\
\text { error }\end{array}$ & $\begin{array}{l}\text { Test Err. } \\
\text { (Standard } \\
\text { Error) }\end{array}$ \\
\hline $\begin{array}{l}\text { KM-G RRFE (NR) } \\
\text { KM-G GRFE (NR) } \\
\text { KM-G RRFE (CP) } \\
\text { Lin Reg Lasso } \\
\text { No Selection }\end{array}$ & $\begin{array}{c}2 \\
0 \\
2 \\
0 \\
\text { NA }\end{array}$ & $\begin{array}{c}67 \\
0 \\
67 \\
0 \\
\text { NA }\end{array}$ & $\begin{array}{c}31 \\
100 \\
31 \\
100 \\
\text { NA }\end{array}$ & $\begin{array}{c}10.55(2.09) \\
8.53(1.97) \\
9.46(1.74) \\
8.49(1.20) \\
\end{array}$ & $\begin{array}{c}7 \\
0 \\
7 \\
0 \\
\text { NA }\end{array}$ & $\begin{array}{c}87 \\
3 \\
87 \\
0 \\
\text { NA }\end{array}$ & $\begin{array}{c}6 \\
97 \\
6 \\
100 \\
\text { NA }\end{array}$ & $\begin{array}{l}7.45(1.51) \\
9.70(1.39) \\
7.87(1.61) \\
8.74(0.90) \\
8.02(0.68)\end{array}$ & $\begin{array}{c}29 \\
0 \\
29 \\
0 \\
\text { NA }\end{array}$ & $\begin{array}{c}67 \\
0 \\
67 \\
0 \\
\text { NA }\end{array}$ & $\begin{array}{c}4 \\
100 \\
4 \\
100 \\
\text { NA } \\
\end{array}$ & $\begin{array}{l}5.72(1.19) \\
9.12(0.96) \\
6.65(1.50) \\
8.75(0.68) \\
7.83(0.47)\end{array}$ & \begin{tabular}{|c}
56 \\
0 \\
56 \\
0 \\
NA
\end{tabular} & $\begin{array}{c}44 \\
4 \\
44 \\
0 \\
\text { NA } \\
\end{array}$ & $\begin{array}{c}0 \\
96 \\
0 \\
100 \\
\text { NA }\end{array}$ & $\begin{array}{l}4.21(0.89) \\
8.36(0.80) \\
5.29(1.04) \\
8.63(0.50) \\
7.23(0.27)\end{array}$ \\
\hline
\end{tabular}

TABLE S5.2. Comparison in regression between (a) KM-G RRFE (NR)-risk-RFE with

KM Gauss with naive ranks, (b) KM-G GRFE (NR)-Guyon RFE with KM Gauss with naive ranks, (c) KM-G RRFE (CP)- risk-RFE with KM Gauss with change point model,

(e) Lin Reg Lasso- linear regression with LASSO, and (f) No Selection- KM Gauss

without selection under nonlinearity, without and with the presence of colinearity.

dominance of RRFE increases with dimension size of the design. Also, performance of GRFE in regression is quite poor, and in higher dimensions, it struggles to find any signals. Unless GRFE-NR and RRFE-NR obtains the exact same performance in feature selection, the misclassification rates in classification and the average test error rates in regression are consistently lower for RRFE-NR than that for GRFE-NR. We also see that in classifi- 
cation, the RRFE-CP procedure yields higher misclassification error rates than both the NR procedures. This is understandable as the NR methods depend on knowing the oracle number of relevant features beforehand (which is never possible to know in practice, and even if it were known, it is clear that RRFE-NR should be the recommended method in that case), while the $\mathrm{CP}$ method, as mentioned before, tends to choose more features than the actual number of true signals on average, thus resulting in accumulation of some noise in the model. In regression however, this effect is not as pronounced as in classification.

In a relative comparison of test error rates, RRFE-NR still dominates RRFE-CP in most cases, but RRFE-CP is seen to do slightly better than RRFE-NR in lower sample sizes, especially when both methods struggle to perform well in feature selection. The RRFE-CP procedure is also seen to dominate GGRE-NR in terms of test error rates more often than not, especially when the latter is struggling in terms of feature selection. The linear selection methods - SVM-SCAD and logistic regression with LASSO in classification and linear regression with LASSO in regression perform very poorly in all situations of the simulation exercise, both in terms of feature selection, and in terms of misclassification or average test error rates. The performance of RRFE-CP (which does feature selection without knowledge of any oracle) is far superior to any of them. Curiously, in some of the settings, SCAD seems to perform reasonably well in feature selection, although it did poorly in terms of misclassification error. This is because the regularization parameter chosen via cross validation for SCAD in those cases allowed selection of almost all of the features, which meant it could retain all/some of the signals a few of the times, but at the expense of increased noise in the model. According to the test set performances of the normal $\mathrm{KM}$ procedure without selection, it is clear why a method such as risk-RFE is useful for feature selection in the nonlinear space, because as seen often, using a linear method here can be worse than using no selection. The no selection procedure yields better test error rates than the RRFE procedure in the case $d=100, d_{0}=4$, for the smallest sample size employed here $n=100$, probably because the RRFE procedures seem to struggle in this setting. This effect disappears when the sample size is increased to $n=200$, and beyond. We see that in classification risk-RFE performs quite similarly when we have colinearity in covariates versus when we do not. In regression, however, it tends to do better when there is no colinearity, and this effect is most pronounced when dimension (and signal) sizes increase (as seen in the last example $\left.d=100, d_{0}=4\right)$. Overall we conclude that in moderately high dimensional classification and regression, when there is enough suspi- 
cion that the underlying structure is not linear, using risk RFE is definitely a good option.

S6. Simulations II: Performance of risk-RFE in the linear space. In this section, we evaluate the scope of the risk-RFE algorithm with kernel machines, when the true underlying data-generating model is linear. We again consider two different data-generating mechanisms, one in classification, and the other in regression. We look at four different scenarios (a) total number of covariates is 15 of which 4 are relevant, (b) 30 covariates with 7 relevant, (c) 50 covariates with 3 relevant, (d) 200 covariates with 10 relevant.

For classification, we consider the hinge loss $L_{H L}$ as the surrogate replacement of the $0-1$ loss, and the KM function is computed using the Gaussian RBF kernel as well as the linear kernel. Covariates $X$ are generated uniformly on $[-1,1]$, and the output $Y$ is associated through a linear relationship with the covariate space $X$ given as $Y=\operatorname{sign}\left(\omega^{\prime} X\right)$, where $\omega$ is the coefficient vector with only the first few elements being non-zero (corresponding to the relevant features chosen at random from a list of coefficients) and the remaining are zero. The effect size of the relevant features are fixed to be either 0.5 or 1 except for the classification example in the high dimensional setting of $d=200, d_{0}=10$, where we enhance them by $50 \%$ to obtain more meaningful comparisons. Again, we initialize the original KM function using 5-fold cross validation on the kernel width $\gamma$ and the regularization parameter $\lambda$ which are chosen from the set of values $\left(\frac{2}{n \lambda}, \gamma\right)=\left(0.01 \times 10^{i}, j\right), i=\{0,1,2,3,4,5\}, j=\{1,2,3,4,5\}$, where $n$ is the sample size for the given setting. We use the linear kernel $k\left(x_{1}, x_{2}\right)=\left\langle x_{1}, x_{2}\right\rangle$ with the $\epsilon$-insensitive $\operatorname{loss} L_{\epsilon}(x, y, f(x))=\max \{0,|y-f(x)|-\epsilon\}$ to treat the regression setting. As before, the value of $\epsilon$ was fixed at 0.1. Covariates are generated as before and $Y$ is still associated linearly with $X$, generated as $Y=\omega^{\prime} X+\frac{1}{3} N_{\operatorname{dim}(X)}(0,1)$. As before we initialize with a 5 -fold cross validation on $\lambda$. We repeat the procedures for different sample sizes $n=\{100,200,400\}$. We also repeat each simulation 100 times. As in the nonlinear setup, we compare the performance of risk-RFE with that of Guyon's RFE to see if we indeed have enhanced performance in consistency of feature selection. We also compare it with other popular methods of feature selection in linear methods, namely SCAD SVM (linear KM with SCAD penalty) and logistic regression with LASSO in classification, and with linear regression with LASSO in regression. We present our results in Table S6.1 (classification) and Table S6.2 (regression). As in the nonlinear setup, we look at the percentage of times the algorithms (a) made no errors, (b) 


\begin{tabular}{|c|c|c|c|c|c|c|c|c|c|c|c|c|}
\hline \multirow{2}{*}{$\begin{array}{l}\text { Classification } \\
d=15, d_{0}=4\end{array}$} & \multicolumn{4}{|c|}{$n=100$} & \multicolumn{4}{|c|}{$n=200$} & \multicolumn{4}{|c|}{$n=400$} \\
\hline & $\begin{array}{l}\% \text { no } \\
\text { error }\end{array}$ & $\begin{array}{l}\% 1 \\
\text { error }\end{array}$ & $\begin{array}{l}\%>1 \\
\text { error }\end{array}$ & $\begin{array}{c}\text { Mean } \\
\text { Test Err }\end{array}$ & $\begin{array}{l}\% \text { no } \\
\text { error }\end{array}$ & $\begin{array}{l}\text { \% one } \\
\text { error }\end{array}$ & $\begin{array}{l}\%>1 \\
\text { error }\end{array}$ & $\begin{array}{c}\text { Mean } \\
\text { Test Err }\end{array}$ & $\begin{array}{l}\% \text { no } \\
\text { error }\end{array}$ & $\begin{array}{l}\% 1 \\
\text { error }\end{array}$ & $\begin{array}{l}\%>1 \\
\text { error }\end{array}$ & $\begin{array}{c}\text { Mean } \\
\text { Test Err }\end{array}$ \\
\hline RFE (NR) & 97 & 3 & 0 & 0.05 & 100 & 0 & 0 & 0.03 & 100 & 0 & 0 & 0.02 \\
\hline KM-L RRFE (NR) & 99 & 1 & 0 & 0.04 & 100 & 0 & 0 & 0.02 & 100 & 0 & 0 & 0.01 \\
\hline 1-G GRFE & 17 & 38 & 45 & 0.20 & 19 & 32 & 49 & 0.21 & 32 & 21 & 47 & 0.18 \\
\hline RFE & 36 & 41 & 23 & 0.16 & 61 & 27 & 12 & 0.11 & 77 & 18 & 5 & 0.07 \\
\hline SVM & 98 & 2 & 0 & 0.04 & 100 & 0 & 0 & 0.02 & 100 & 0 & 0 & 0.01 \\
\hline so & 94 & 6 & 0 & 0.03 & 100 & 0 & 0 & 0.01 & 100 & 0 & 0 & 0.01 \\
\hline$d=30, d_{0}=7$ & $\begin{array}{l}\% \text { no } \\
\text { error }\end{array}$ & $\begin{array}{l}\% 1 \\
\text { error }\end{array}$ & $\begin{array}{l}\%>1 \\
\text { error }\end{array}$ & $\begin{array}{c}\text { Mean } \\
\text { Test Err }\end{array}$ & $\begin{array}{l}\% \text { no } \\
\text { error }\end{array}$ & $\begin{array}{l}\% \text { one } \\
\text { error }\end{array}$ & $\begin{array}{l}\%>1 \\
\text { error }\end{array}$ & $\begin{array}{c}\text { Mean } \\
\text { Test Err }\end{array}$ & $\begin{array}{l}\% \text { no } \\
\text { error }\end{array}$ & & $\begin{array}{l}0>1 \\
\text { rrror }\end{array}$ & $\begin{array}{c}\text { Mean } \\
\text { Test Err }\end{array}$ \\
\hline RFE & 62 & 34 & 4 & 0.09 & 100 & 0 & 0 & 0.04 & 100 & 0 & 0 & 0.03 \\
\hline KM-L RRFE & 72 & 24 & 4 & & 100 & 0 & 0 & 0.03 & 100 & 0 & 0 & 0.02 \\
\hline G GRFE & 1 & 7 & 92 & 0.24 & 10 & 9 & 81 & 0.26 & 2 & 6 & 92 & 0.26 \\
\hline RFE & 10 & 24 & 75 & 0.23 & 23 & 36 & 41 & 0.16 & 55 & 32 & 13 & 0.10 \\
\hline & 83 & 14 & 3 & 0.08 & 100 & 0 & 0 & & 100 & 0 & 0 & 0.02 \\
\hline Log Reg Lasso & 46 & 49 & 5 & 0.09 & 96 & 4 & 0 & 0.02 & 100 & 0 & 0 & 0.01 \\
\hline$d=50, d_{0}=3$ & $\begin{array}{l}\% \text { no } \\
\text { error }\end{array}$ & $\begin{array}{l}\% 1 \\
\text { error }\end{array}$ & $\%>$ & $\begin{array}{c}\text { Mean } \\
\text { Test Err }\end{array}$ & $\begin{array}{l}\% \text { no } \\
\text { error }\end{array}$ & $\begin{array}{l}\% \text { o } \\
\text { err }\end{array}$ & $\begin{array}{l}\% \\
\text { err }\end{array}$ & $\begin{array}{l}\text { Mean } \\
\text { est Err }\end{array}$ & $\begin{array}{l}\% \text { no } \\
\text { error }\end{array}$ & & $\begin{array}{l}\%>1 \\
\text { error }\end{array}$ & $\begin{array}{c}\text { Mean } \\
\text { Test Err }\end{array}$ \\
\hline KM-G RRFE & 95 & 5 & 0 & 5 & 100 & 0 & 0 & 0 . & 100 & 0 & 0 & 0.02 \\
\hline $\mathrm{RFE}(\mathrm{NR})$ & 94 & 6 & 0 & 0.06 & 100 & 0 & 0 & 0.03 & 100 & 0 & 0 & 0.01 \\
\hline $\mathrm{E}$ & 29 & 54 & 17 & 0.14 & 41 & 55 & 4 & 0.10 & 37 & 58 & 5 & 0.10 \\
\hline $\mathrm{E}$ & 41 & 30 & 29 & 0.20 & 59 & 27 & 14 & 0 . & 89 & 9 & 2 & 0.04 \\
\hline & 98 & 2 & 0 & 0.06 & 100 & 0 & 0 & 0.03 & 100 & 0 & 0 & 0.01 \\
\hline & 97 & 3 & 0 & 0.02 & 100 & 0 & 0 & 0.01 & 100 & 0 & 0 & 0.01 \\
\hline$d=200, d_{0}=10$ & $\begin{array}{l}\% \text { no } \\
\text { error }\end{array}$ & $\begin{array}{l}\% 1 \\
\text { error }\end{array}$ & $\begin{array}{l}\%> \\
\text { erro }\end{array}$ & $\begin{array}{c}\text { Mean } \\
\text { Test Err }\end{array}$ & $\begin{array}{l}\% \text { no } \\
\text { error }\end{array}$ & $\begin{array}{ll}\% & 0 \\
\text { erre }\end{array}$ & $\begin{array}{l}\% \\
\text { err }\end{array}$ & $\begin{array}{c}\text { Mean } \\
\text { Test Err }\end{array}$ & $\begin{array}{l}\% \text { no } \\
\text { error }\end{array}$ & $\%$ & $\begin{array}{l}\%>1 \\
\text { error }\end{array}$ & $\begin{array}{c}\text { Mean } \\
\text { Test Err }\end{array}$ \\
\hline $\mathrm{E}(\mathrm{NR})$ & 5 & 12 & 83 & 27 & 72 & 24 & 4 & 7 & 100 & 0 & 0 & 0.04 \\
\hline KM-G RRFE (CP) & 10 & 25 & 65 & & 76 & 21 & 3 & & 100 & 0 & 0 & 0.02 \\
\hline RFE (NR) & 0 & 6 & 94 & 0.26 & 34 & 33 & 33 & 0.13 & 97 & 3 & 0 & 0.05 \\
\hline $2 \mathrm{FE}(\mathrm{CP})$ & 11 & 29 & 60 & 0.25 & 56 & 37 & 7 & 0.10 & 100 & 0 & 0 & 0.03 \\
\hline & 0 & 0 & 100 & 0.42 & 0 & 0 & 100 & 0.40 & 2 & 3 & 95 & 0.36 \\
\hline $2 F E$ & 0 & 6 & 94 & 0.26 & 30 & 39 & 31 & 0.15 & 96 & 4 & 0 & 0.09 \\
\hline & 3 & 18 & 79 & & 69 & 26 & 5 & & 100 & 0 & 0 & 0.05 \\
\hline Log Reg Lasso & 0 & 2 & 98 & 0.21 & 42 & 45 & 13 & 0.08 & 99 & 1 & 0 & 0.02 \\
\hline
\end{tabular}

TABLE S6.1. Comparison between (a) KM-G RRFE - risk-RFE with KM Gauss, (b) KM-L RRFE - risk-RFE with KM Linear, (c) KM-G GRFE - Guyon RFE with KM Gauss (d) SCAD SVM - KM Linear (SVM) with SCAD (e) Log Reg Lasso - Logistic regression with LASSO in a classification setting (' $N R$ ' denotes using naive ranks, ' $C P$ ' denotes using change point method) under linearity

made only one error, or (c) made multiple errors, and also note the average test prediction error.

For the penalized methods, we use an ad-hoc cross validation technique to select the minimum amount of penalization that would result in retaining at least as many features as there are important features in the model. However, in the high dimensional case with $n / d=1 / 2\left(d=200, d_{0}=10, n=100\right)$, we use both the $\mathrm{CP}$ and $\mathrm{NR}$ formulations of risk-RFE to show that using 


\begin{tabular}{|c|c|c|c|c|c|c|c|c|c|c|c|c|}
\hline Regression & & & $=100$ & & & & $=200$ & & & & $=400$ & \\
\hline$d=15, d_{0}=4$ & $\begin{array}{l}\% \text { no } \\
\text { error }\end{array}$ & $\begin{array}{l}\% 1 \\
\text { error }\end{array}$ & $\begin{array}{l}\%>1 \\
\text { error }\end{array}$ & $\begin{array}{c}\text { Mean } \\
\text { Test Err }\end{array}$ & $\begin{array}{l}\% \text { no } \\
\text { error }\end{array}$ & $\begin{array}{l}\% \text { on } \\
\text { error }\end{array}$ & $\begin{array}{l}\%>1 \\
\text { error }\end{array}$ & $\begin{array}{c}\text { Mean } \\
\text { Test Err }\end{array}$ & $\begin{array}{l}\% \text { no } \\
\text { error }\end{array}$ & $\begin{array}{l}\% 1 \\
\text { error }\end{array}$ & $\begin{array}{l}\%>1 \\
\text { error }\end{array}$ & $\begin{array}{c}\text { Mean } \\
\text { Test Err }\end{array}$ \\
\hline KM-L $\mathrm{BRFE}$ & 100 & 0 & 0 & 0.12 & 100 & 0 & 0 & 0.12 & 100 & 0 & 0 & 0.11 \\
\hline FE & 100 & 0 & 0 & 0.12 & 100 & 0 & 0 & 0.12 & 100 & 0 & 0 & 0.11 \\
\hline $\mathrm{SSC}$ & 100 & 0 & 0 & 0.12 & 100 & 0 & 0 & 0.12 & 100 & 0 & 0 & 0.11 \\
\hline$d=30, d_{0}=7$ & $\begin{array}{l}\% \mathrm{n} \\
\text { erro }\end{array}$ & $\begin{array}{l}\% \\
\text { err }\end{array}$ & $>1$ & $\begin{array}{c}\text { Mean } \\
\text { Test Err }\end{array}$ & $\begin{array}{l}\% \mathrm{n} \\
\mathrm{errc}\end{array}$ & 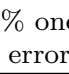 & $\begin{array}{l}\%>1 \\
\text { error }\end{array}$ & $\begin{array}{l}\text { Mean } \\
\text { Test Err }\end{array}$ & $\begin{array}{l}\% \text { no } \\
\text { error }\end{array}$ & $\%$ & $\begin{array}{l}0>1 \\
\text { error }\end{array}$ & $\begin{array}{c}\text { Mean } \\
\text { Test Err }\end{array}$ \\
\hline $\mathrm{KM}$ & 100 & 0 & 0 & 0.13 & 100 & 0 & 0 & 0.12 & 100 & 0 & 0 & 0.11 \\
\hline & 100 & 0 & 0 & 0.13 & 100 & 0 & 0 & 0.12 & 100 & 0 & 0 & 0.11 \\
\hline isso & 99 & 1 & 0 & 0.12 & 100 & 0 & 0 & 0.12 & 100 & 0 & 0 & 0.11 \\
\hline$d=50, d_{0}=3$ & $\begin{array}{l}\% \text { no } \\
\text { error }\end{array}$ & $\begin{array}{l}\% 1 \\
\text { error }\end{array}$ & $\begin{array}{l}\%>1 \\
\text { error }\end{array}$ & $\begin{array}{c}\text { Mean } \\
\text { Test Err }\end{array}$ & $\begin{array}{l}\% \text { no } \\
\text { error }\end{array}$ & $\begin{array}{l}\text { \% on } \\
\text { error }\end{array}$ & $\begin{array}{l}\%>1 \\
\text { error }\end{array}$ & $\begin{array}{c}\text { Mean } \\
\text { Test Err }\end{array}$ & $\begin{array}{l}\% \text { no } \\
\text { error }\end{array}$ & $\begin{array}{l}\% 1 \\
\text { error }\end{array}$ & $\begin{array}{l}\%>1 \\
\text { error }\end{array}$ & $\begin{array}{c}\text { Mean } \\
\text { Test Err }\end{array}$ \\
\hline KM-L RRFE & 100 & 0 & 0 & 0.13 & 100 & 0 & 0 & 0.11 & 100 & 0 & 0 & 0.11 \\
\hline GRFE & 100 & 0 & 0 & 0.13 & 100 & 0 & 0 & 0.11 & 100 & 0 & 0 & 0.11 \\
\hline Lin Reg Lasso & 100 & 0 & 0 & 0.12 & 100 & 0 & 0 & 0.11 & 100 & 0 & 0 & 0.11 \\
\hline$d=200, d_{0}=10$ & $\begin{array}{l}\% \text { no } \\
\text { error }\end{array}$ & $\begin{array}{l}\% 1 \\
\text { error }\end{array}$ & $\begin{array}{l}\%>1 \\
\text { error }\end{array}$ & $\begin{array}{c}\text { Mean } \\
\text { Test Err }\end{array}$ & $\begin{array}{l}\% \text { no } \\
\text { error }\end{array}$ & $\begin{array}{l}\% \text { ol } \\
\text { erro }\end{array}$ & $\begin{array}{l}\%>1 \\
\text { error }\end{array}$ & $\begin{array}{c}\text { Mean } \\
\text { Test Err }\end{array}$ & $\begin{array}{l}\% \text { no } \\
\text { error }\end{array}$ & $\begin{array}{l}\% 1 \\
\text { error }\end{array}$ & $\begin{array}{l}\%>1 \\
\text { error }\end{array}$ & $\begin{array}{c}\text { Mean } \\
\text { Test Err }\end{array}$ \\
\hline KM-L I & 80 & 14 & 6 & 0.22 & 100 & 0 & 0 & 0.13 & 100 & 0 & 0 & 0.12 \\
\hline KM-I & 81 & 14 & 5 & 0.2 & 100 & 0 & 0 & 0.13 & 100 & 0 & 0 & 0.12 \\
\hline Lin Reg Lasso & 49 & 31 & 20 & 0.24 & 98 & 2 & 0 & 0.12 & 100 & 0 & 0 & 0.11 \\
\hline
\end{tabular}

TABLE S6.2. Comparison between (a) KM-L RRFE - risk-RFE with KM Linear, (b) KM-L GRFE - Guyon RFE with KM Linear (c) Lin Reg Lasso - Linear ergression with LASSO in a regression setting (only ' $N R$ ' was used) under linearity

the CP model can potentially do better at feature selection than using just $\mathrm{NR}$, as the change point (CP) model can allow for better retention of signals at the cost of some additional noise in the model (see Figure 1).

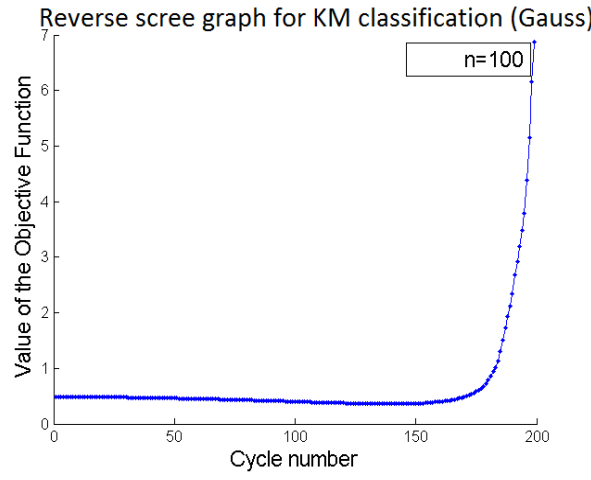

FIG 1. Reverse scree graph of the objective function values for one simulation run in $d=200, d_{0}=10$ for KM classification with Gaussian kernel.

Since the underlying relationship between $Y$ and the features $X$ is linear in 
each of the simulated examples in this section, we expect penalized methods to perform at least as well as risk-RFE in these settings. For the regression example, as can be seen in Table S6.2, all methods are performing equally well in the lower dimensional settings, while the LASSO is actually doing worse than both risk-RFE and Guyon's RFE when $n$ was small in the high dimensional setting $\left(d=200, d_{0}=10\right)$. In the classification setting (see Table S6.1), Guyon's RFE is performing very poorly even when the sample size is high (using a linear kernel only improves the performance somewhat, but it is still quite poor), which justifies the need for the modification to have more consistent performances than what we see from risk-RFE. Risk-RFE is dominating the performance of the LASSO as well, more so in the smaller sample sizes, while in larger sample sizes both are performing equally well. Unsurprisingly, SCAD SVM is showing the best comparative performance here. Risk-RFE performs somewhat poorly in comparison to SCAD only in the setting $d=30, d_{0}=7, n=100$, while it does equally well as SCAD in all other settings. In fact, in the high dimensional setting $\left(d=200, d_{0}=10\right)$ when $n=100$, using the change point method allowed risk-RFE to pick out signals with at most one mistake about $40 \%$ of the time, compared to $21 \%$ of the time that SCAD was able to do so. On further inspection of this setting, it was seen that when the CP method was used, risk-RFE allowed at most 5 mistakes overall (2-5 mistakes the remaining $60 \%$ of the time). Risk-RFE with NR is actually doing similarly as SCAD SVM in this regard (both made 2-5 errors about $70 \%$ of the time) in this scenario.

A look at Table S6.1 reveals that although logistic regression with LASSO is not always the best performing feature selection method, it still obtains low misclassification error (in cases where its feature selection performance actually improves). This is probably due to the fact that the underlying relationships are all linear (in contrast to results in the nonlinear setup where it obtains very poor misclassification error), an effect the linear KM couldn't replicate (as can be seen from errors obtained from the SCAD SVM and risk-RFE with linear KM). Overall we can conclude from the above that risk-RFE is doing very well when the underlying relationship between the input space and the output is linear, even when compared with some of well known high performing linear methods such as the regression LASSO or SCAD-SVM.

S7. Example applications: Additional details. We apply the riskRFE algorithm to three feature selection applications: feature selection in vowel recognition data, feature selection in predicting total UPDRS (unified Parkinson's disease rating scale) scores in people with early-stage Parkin- 
son's disease, and feature selection in predicting 'Per Capita Violent Crimes' in the Crimes dataset.

S7.1. Non-linear classification in vowel recognition. To show the applicability of RFE for non-linear classification, we apply our method to the vowel recognition data set available at http://www-stat.stanford.edu/ tibs/ ElemStatLearn/ [see Hastie, Tibshirani and Friedman, 2009]. The dataset consists of recordings from 15 individuals speaking different classes of vowels, 11 in all, and a 10 dimensional input space created from the reflection coefficients to calculate 10 log area parameters [see Rabiner and Schafer, 1978]. We apply KM with a Gaussian RBF kernel to classify between the vowel sounds ' $\mathrm{i}$ ' and ' $\mathrm{I}$ ' based on these 10 features. We use five-fold cross-validation to obtain the KM parameters, and it is then trained on a data set with 48 instances and tested with 42 instances of each vowel. We compared results of running risk-RFE with KM Gaussian (KM Gauss-wRFE), with (a) running $\mathrm{KM}$ without any feature selection (KM Gauss-woRFE), (b) running logistic regression with LASSO and (c) SCAD SVM (linear KM with SCAD). We did not use Guyon's RFE as it does not provide any inherent mechanism of choosing a subset of features. As can be seen in Table 6.1 in Section 7, risk-RFE is able to achieve a more than $40 \%$ drop in misclassification error than its nearest competitor.

S7.2. Non linear regression in Parkinsons data. This dataset can be found at https://archive.ics.uci.edu/ml/datasets/Parkinsons+ Telemonitoring and is composed of a range of biomedical voice measurements from 42 people with early-stage Parkinson's disease recruited for a six-month trial of a telemonitoring device [See Tsanas et al., 2010, for more details]. There were 20 covariates in all, including the subject id, age, gender, time interval from baseline recruitment date and 16 biomedical voice measures. Each data instance corresponds to one of 5,875 voice recordings from these individuals. One of the main aims of the data was to predict the motor UPDRS score from the 16 voice measures. We split the data randomly into ten test and training sets of equal sizes and perform KM regression with an epsilon-insensitive loss and Gaussian RBF kernel on each training-test data pair. Five-fold cross-validation was used to select the parameters of the algorithm in each run. The risk-RFE algorithm selects the same 3 variables (the subject id, age and the time interval from baseline recruitment date) in each run, achieving an average test error of 10.08 units. We also compared results from (i) when we run KM regression without using risk-RFE, and (ii) when we use linear regression with LASSO for feature selection. While the standard KM algorithm uses all 20 features and obtains a test error of 
11.02 units, LASSO performs poorly choosing an average of 17.4 features with a test error of 87.80 units, owing probably to the fact that the underlying relationship is nonlinear (see Table 7.1 in Section 7). Thus, the risk-RFE algorithm not only helps build a more parsimonious regression model with better test prediction abilities, but it also identified the redundancy of the 16 voice measures in predicting UPDRS scores.

S7.3. Non linear regression in Crimes data. The Crimes dataset was compiled by Michael Redmond at La Salle University, and can be found at https://archive.ics.uci.edu/ml/datasets/Communities+ and+Crime. This dataset combines socio-economic data from the 1990 US Census, law enforcement data from the 1990 US Law Enforcement Management and Administrative Statistics (LEMAS) Survey, and crime data from the 1995 FBI Uniform Crime Report (UCR). The full dataset contains crime information in 1994 communities through 128 socio-economic variables relating to community and law enforcement. The goal of learning here is to predict 'Per Capita Violent Crimes', which is a crime score compiled for each community, based on the remaining attributes. Owing to missingness in some of the covariates, they were removed from the analysis for simplicity. The final task consisted of building a regression model for 'Per Capita Violent Crimes' based on data from complete information from 101 features on 1993 communities. As before, we split the data randomly into ten test and training sets of equal sizes and perform KM regression with epsiloninsensitive loss and Gaussian RBF kernel on each training-test data pair. Five-fold cross-validation was used to select the parameters of the algorithm in each run. The risk-RFE algorithm selects only 11 features on average and achieves a test error of 0.02 units, the same as when i) we run the KM regression algorithm without using risk-RFE, or ii) when we use linear regression with LASSO for feature selection. LASSO is performing as well as the nonlinear KM regression algorithm in this case unlike the first regression example, probably owing to the fact that the underlying relationship is likely linear. It is worthwhile to note that even if the risk-RFE algorithm does not show any improvement in performance with respect to test error rates, it selects only 11 features out of 101 (only 11\%), while LASSO selects on an average around 38 features, and the normal KM procedure takes into account all 101 features in forming the decision function (see Table 7.1 in Section 7). Obtaining a parsimonious model can be as important as finding a model with good test error performance. Here, using risk-RFE allows us to obtain a much simpler model with equivalent error performance relative to the other methods. 


\section{S8. Proofs of results from the main draft.}

\section{S8.1. Proof of Lemma 3 (from Section 3.2.1).}

Proof. To see this, let us consider a dot-product kernel $k$ such that $k(x, y)=g(\langle x, y\rangle)$ where $\langle\cdot, \cdot\rangle$ is the usual Euclidean inner-product. Now consider the pre-RKHSs $H_{\text {pre }}$ and $H_{\text {pre }}^{J}$. We show here that $H_{\text {pre }}^{J} \subseteq H_{\text {pre }}$ which will imply that $H^{J} \subseteq H$. To show this, take $f \in H_{\text {pre }}^{J}$ This implies that $f$ can be written as $f(\cdot)=\sum_{i=1}^{n} \alpha_{i} k^{J}\left(\cdot, x_{i}\right)$ for $n \in \mathbb{N}, \alpha_{1}, \ldots, \alpha_{n} \in$ $\mathbb{R}, x_{1}, \ldots, x_{n} \in \mathcal{X}$. Hence,

$$
\begin{aligned}
f(\cdot) & =\sum_{i=1}^{n} \alpha_{i} k^{J}\left(\cdot, x_{i}\right)=\sum_{i=1}^{n} \alpha_{i} k\left(\pi^{J^{c}}(\cdot), \pi^{J^{c}}\left(x_{i}\right)\right) \\
& =\sum_{i=1}^{n} \alpha_{i} g\left(\left\langle\pi^{J^{c}}(\cdot), \pi^{J^{c}}\left(x_{i}\right)\right\rangle\right)=\sum_{i=1}^{n} \alpha_{i} g\left(\left\langle\cdot, \pi^{J^{c}}\left(x_{i}\right)\right\rangle\right) \\
& =\sum_{i=1}^{n} \alpha_{i} k\left(\cdot, \pi^{J^{c}}\left(x_{i}\right)\right)
\end{aligned}
$$

Noting that $\pi^{J^{c}}\left(x_{1}\right), \ldots, \pi^{J^{c}}\left(x_{n}\right) \in \mathcal{X}$, we have that $f \in H_{\text {pre. In a similar }}$ way, we can show that for any $J_{1} \subseteq J_{2}, H^{J_{2}} \subseteq H^{J_{1}}$.

\section{S8.2. Proof of Lemma 4 (from Section 4).}

Proof. (i) First recall notational definitions (10) from Section 4.1. Now fixing a $\lambda \in[0,1]$, we have that $B:=c_{L}\left(\lambda^{-1 / 2}\right) \lambda^{-1 / 2}+1 \leq 2 \lambda^{-1 / 2}$. Now since $|X| \leq x \Rightarrow X \leq x$ for any $x \geq 0$, we see from Proposition S5 in the Supplementary Section S3 that for $J_{1}, J_{2} \in \widetilde{\mathcal{J}}$

$\mathcal{R R}_{D}^{D}\left(J_{2}, J_{1}\right)<A_{2}{ }^{J_{1}}(\lambda)+A_{2}^{J_{2}}(\lambda)+24 \lambda^{-1 / 2} \sqrt{\frac{2 \tau}{n}}+40 \lambda^{-1 / 2} \frac{\tau}{n}+48 K_{2} \lambda^{-\frac{p-1}{2}}\left(\frac{a^{2 p}}{\lambda^{p} n}\right)^{\frac{1}{2}}$

$$
=A_{2}^{J_{1}}(\lambda)+A_{2}^{J_{2}}(\lambda)+24 \sqrt{2 \tau}(\lambda n)^{-\frac{1}{2}}+40 \tau\left(\lambda^{\frac{1}{2}} n\right)^{-1}+48 K_{2} a^{2 p}(\lambda n)^{-\frac{1}{2}}
$$

with probability at least $1-2 e^{-\tau}$. Now since $\lambda_{n} \rightarrow 0$ and $\lim _{n \rightarrow \infty} \lambda_{n} n=\infty$, Lemma 5.15 along with (5.32) of SC08 gives us that the right hand side of the above inequality converges to 0 . To establish the convergence rate of our algorithm, we will now make use of the bound on $A_{2}{ }^{J}(\lambda)$ assumed in (RC1) (also see Remark 17). It can be now seen that asymptotically the best choice for $\lambda_{n}$ in (3) is a sequence that behaves as $n^{-\frac{1}{(2 \beta+1)}}$ and then the 
inequality in (3) is satisfied with the l.h.s. replaced by $\epsilon_{n}$, where $\epsilon_{n}$ is given by $\left(2 c+24 \sqrt{2 \tau}+48 K_{2} a^{2 p}\right) n^{-\frac{\beta}{2 \beta+1}}+40 \tau n^{-\frac{4 \beta+1}{2(2 \beta+1)}}$. This proves (i) for $\left\{\epsilon_{n}\right\}$ for $\tau=o\left(n^{\frac{2 \beta}{2 \beta+1}}\right)$.

(ii) Note that from Corollary S6 in the Supplementary Section S3 and arguments in the proof of (i) given above, for any $J \in\{1,2, \ldots, d\}$ we have

$$
\left|\mathcal{R R}_{D}^{D}(J)\right|<A_{2}^{J}(\lambda)+12 \sqrt{2 \tau}(\lambda n)^{-\frac{1}{2}}+20 \tau\left(\lambda^{\frac{1}{2}} n\right)^{-1}+24 K_{2} a^{2 p}(\lambda n)^{-\frac{1}{2}}=\epsilon_{n} / 2
$$

with probability at least $1-e^{-\tau}$. Now note that from Assumption (A2) in Section 3.4, for $J_{1} \in \tilde{\mathcal{J}}, J_{2} \notin \tilde{\mathcal{J}}$, we have that $\mathcal{R}^{*}{ }_{L, P, H^{J_{2}}}-\epsilon_{0} \geq \mathcal{R}^{*}{ }_{L, P, H^{J_{*}}}$ $=\mathcal{R}^{*}{ }_{L, P, H^{J_{1}}}$. So for $H^{J_{2}}$ we have,

$$
\begin{aligned}
& P^{n}\left(\left|\mathcal{R R}_{D}^{D}\left(J_{2}\right)\right|<\epsilon_{n} / 2\right)>1-e^{-\tau} \\
\Rightarrow & P^{n}\left(\mathcal{R}_{L, D, H^{J_{2}}}^{\mathrm{reg}, \lambda_{n}}\left(f_{D, \lambda_{n}, H^{J_{2}}}\right)+\epsilon_{n} / 2>\mathcal{R}^{*}{ }_{L, P, H^{J_{2}}}\right)>1-e^{-\tau},
\end{aligned}
$$

and for $H^{J_{1}}$ we have

$$
\begin{aligned}
& P^{n}\left(\left|\mathcal{R}_{\mathcal{R}_{D}^{D}}^{D}\left(J_{1}\right)\right|<\epsilon_{n} / 2\right)>1-e^{-\tau} \\
\Rightarrow & P^{n}\left(\mathcal{R}_{L, D, H^{J_{1}}}^{\mathrm{reg}, \lambda_{n}}\left(f_{D, \lambda_{n}, H^{J_{1}}}\right)<\mathcal{R}^{*}{ }_{L, P, H^{J_{1}}}+\epsilon_{n} / 2\right)>1-e^{-\tau} \\
\Rightarrow & P^{n}\left(\mathcal{R}_{L, D, H^{J_{1}}}^{\mathrm{reg}, \lambda_{n}}\left(f_{D, \lambda_{n}, H^{J_{1}}}\right)+\epsilon_{0}-\epsilon_{n} / 2<\mathcal{R}^{*}{ }_{L, P, H^{J_{2}}}\right)>1-e^{-\tau} .
\end{aligned}
$$

The above two statements then jointly imply that

$$
\mathcal{R R}_{D}^{D}\left(J_{2}, J_{1}\right)>\epsilon_{0}-\epsilon_{n}
$$

with $P^{n}$ probability greater than $1-2 e^{-\tau}$. Also it is easy to see that since $\epsilon_{n} \rightarrow 0$ with $n \rightarrow \infty$ for $\tau=o\left(n^{\frac{2 \beta}{2 \beta+1}}\right)$, the gap $\widetilde{\epsilon}_{n}=\epsilon_{0}-\epsilon_{n} \longrightarrow \epsilon_{0}>0$.

(iii) From assumption (A1) in Section 3.3 we have $\mathcal{R}^{*}{ }_{L, P, H}=\mathcal{R}^{*}{ }_{L, P, H^{J}}$ for $J \in \tilde{\mathcal{J}}$. Now using Corollary S7 in the Supplementary Section S3, conditions on $\lambda_{n}, A_{2}{ }^{J}\left(\lambda_{n}\right)$, the 'if' condition of (iii) follows for all $\epsilon>0, \tau>0$ and $n \geq 1$, since

$$
P^{n}\left(\left|\mathcal{R}_{L, P, H^{J}}^{\mathrm{reg}, \lambda_{n}}\left(f_{D, \lambda_{n}, H^{J}}\right)-\mathcal{R}^{*}{ }_{L, P, H}\right|<\eta_{n}\right)>1-e^{-\tau},
$$

where $\eta_{n}=\left(c+8 \sqrt{2 \tau}+16 K_{2} a^{2 p}\right) n^{-\frac{\beta}{2 \beta+1}}+40 / 3 \tau n^{-\frac{4 \beta+1}{2(2 \beta+1)}}$.

Now for $J \notin \widetilde{\mathcal{J}}$ we have $\mathcal{R}^{*}{ }_{L, P, H^{J}}-\epsilon_{0} \geq \mathcal{R}^{*}{ }_{L, P, H^{J}}=\mathcal{R}^{*}{ }_{L, P, H}$ and hence the 'only if' condition of (iii) also follows by noting that $\lambda_{n}\left\|f_{D, \lambda_{n}, H^{J}}\right\|_{H^{J}}^{2}+$ $\mathcal{R}_{L, P}\left(f_{D, \lambda_{n}, H^{J}}\right)-\mathcal{R}^{*}{ }_{L, P, H}>\epsilon_{0}-\eta_{n}$ occurs with $P^{n}$ probability greater than $1-e^{-\tau}$. Now since $\eta_{n} \rightarrow 0$ with $n \rightarrow \infty$ and $\tau=o\left(n^{\frac{2 \beta}{2 \beta+1}}\right)$, the gap $\widetilde{\widetilde{\epsilon}}_{n}=\epsilon_{0}-\eta_{n} \longrightarrow \epsilon_{0}>0$. The additional assumption that RKHS $H$ is dense in $\mathcal{L}_{\infty}(\mathcal{X})$ gives us universal consistency for our feature elimination algorithm. 
S8.3. Proof of Lemma 5 (from Section 5.1). In order to prove Lemma 5 for the Gaussian RBF kernel $k_{\gamma}(x, y)=e^{-\gamma^{2}\|x-y\|_{2}^{2}}$, we need to verify regularity conditions $(\mathrm{RC} 0)$ and $(\mathrm{RC} 1)$ from Section 2.6 in this setup. First note that $L_{H L}$ is Lipschitz continuous and bounded for all 3-tuples of the form $(x, y, 0)$ (see Example 2.27 in SC08). Separability of $H_{\gamma}$ holds since an RKHS over a separable metric space having a continuous kernel is separable (Lemma 4.33 of SC08). It is also easy to see that $\left|k_{\gamma}(x, y)\right| \leq 1$ is true for all $x, y \in \mathcal{X}$ and all $\gamma>0$ and hence $\left\|k_{\gamma}\right\|_{\infty} \leq 1$. Instead of showing that entropy condition (6) holds, we will show that the equivalent condition (7) (as discussed in Remark 8) holds here, which will be good enough for our purpose, as can be seen from the steps (9) in the proof of Proposition S9.4 in the Supplementary Section. This is observed by noticing that Theorem 7.34 with Corollary 7.31 of SC08 along with the fact that $d /(d+\tau)$ is an increasing function in $d$, yields a bound as given here with $a:=c_{\epsilon, p} \gamma^{\frac{(1-p)(1+\epsilon) d}{2 p}}$ for all $\gamma \geq 1$, for all $\epsilon>0, d /(d+\tau)<p<1$ and a constant $c_{\epsilon, p}$ depending only on $p$ and a given $\epsilon$, and where $\tau \in(0, \infty]$ is the tail exponent of the distribution $P_{\mathcal{X}}$ as mentioned before [see chapter 7 of $\mathrm{SC08}$, for definition of the tail exponent of a distribution].

Now we need to bound $A_{2}{ }^{J_{*}}(\lambda)$. Note that this can be obtained from Theorem 2.7 in Steinwart and Scovel [2007], where we see that the approximation error for a KM using Gaussian RBF kernel of width $1 / \gamma$ and with number of signals $d_{0}$ can be bounded by $A_{2}\left(\lambda, d_{0}, \gamma\right) \leq c_{d_{0}}\left(\gamma^{d_{0}} \lambda+C\left(2 d_{0}\right)^{\alpha d_{0} / 2} \gamma^{-\alpha d_{0}}\right)$, where $P$ is a distribution on $\mathcal{X}^{J_{*}} \times\{-1,1\} \subset \mathbb{R}^{d_{0}} \times\{-1,1\}, \alpha \in(0, \infty)$ is the geometric noise exponent, $C$ is the constant appearing in Definition 2.2 of Steinwart and Scovel [2007], and $c_{d_{0}}$ is a constant depending only on $d_{0}$. So for a given pair $\left(\lambda, d_{0}\right)$ if we choose $\gamma\left(\lambda, d_{0}\right)=\lambda^{-\frac{1}{(\alpha+1) d_{0}}}$ then it can be seen that $A_{2}\left(\lambda, d_{0}, \gamma\left(\lambda, d_{0}\right)\right) \leq K_{d_{0}} \lambda^{\frac{\alpha}{\alpha+1}}$. So for a sequence of KM objective functions $\lambda_{n}\|f\|_{H_{\gamma\left(\lambda_{n}\right)}}^{2}+\frac{1}{n} \sum_{i=1}^{n} \max \left\{0,1-y_{i} f\left(x_{i}\right)\right\}$ defined for a sequence $\lambda_{n}^{-1}=o(n)$ with $\lambda_{n} \rightarrow 0$ the assumptions for the theoretical results on consistency of risk-RFE are met, and thus Lemma 5 is proved.

\section{S9. Proofs of results from the Supplementary Section.}

\section{S9.1. Proof of Lemma S1.}

Proof. The direction $\mathcal{L}_{\infty}^{J}\left(\mathcal{X}^{J}\right) \subseteq \mathcal{L}_{\infty}\left(\mathcal{X}^{J}\right)$ is obvious since co-ordinate projection maps are continuous. To show that $\mathcal{L}_{\infty}^{J}\left(\mathcal{X}^{J}\right) \supseteq \mathcal{L}_{\infty}\left(\mathcal{X}^{J}\right)$ let us take $g \in \mathcal{L}_{\infty}\left(\mathcal{X}^{J}\right)$. Then $g: \mathcal{X}^{J} \mapsto \mathbb{R}$ is measurable with $\|g\|_{\infty}<\infty$. Extend $g$ to $\widetilde{g}$ to include the whole domain $\mathcal{X}$ by defining $\widetilde{g}(x)=g\left(\pi^{J^{c}}(x)\right)$. Since $\widetilde{g}$ 
is measurable with $\|\widetilde{g}\|_{\infty}=\|g\|_{\infty}$, we have that $\widetilde{g} \in \mathcal{L}_{\infty}(\mathcal{X})$ and $\widetilde{g} \circ \pi^{J^{c}}=\widetilde{g}$, so $g=\left.\widetilde{g}\right|_{\mathcal{X}^{J}} \in \mathcal{L}_{\infty}^{J}\left(\mathcal{X}^{J}\right)$.

\section{S9.2. Proof of Lemma S3.}

Proof. (1) For any function $f \in \mathcal{L}_{\infty}(\mathcal{X})$, by the denseness of $\mathcal{F}$ we can find a sequence of functions $\left\{g_{n}\right\} \in \mathcal{F}$ such that $g_{n} \rightarrow f$ uniformly. Now fix an arbitrary function $f \in \mathcal{L}_{\infty}^{J}(\mathcal{X}) \subset \mathcal{L}_{\infty}(\mathcal{X})$ and consider any sequence of functions $\left\{g_{n}\right\} \in \mathcal{F}$ that converges to $f$ uniformly. Construct the new sequence of functions $\left\{g_{n}^{J}\right\}$ where for any function $f \in \mathcal{F}, f^{J}$ is defined by $f^{J}(x)=f\left(\pi^{J^{c}}(x)\right)$. Observe trivially that $\left\{g_{n}^{J}\right\} \in \mathcal{F}^{J}$.

Now $\left\{g_{n}\right\} \mapsto f$ uniformly $\Rightarrow$ for any $\epsilon>0, \exists N<\infty$ such that $\forall n \geq N$,

$$
\begin{aligned}
& \sup _{x \in \mathcal{X}}\left|g_{n}(x)-f(x)\right|<\epsilon \quad \forall n \geq N \\
\Rightarrow \quad & \sup _{x \in \pi^{J^{c}}(\mathcal{X})}\left|g_{n}(x)-f(x)\right|<\epsilon \quad \forall n \geq N \\
\Rightarrow \quad & \sup _{x \in \mathcal{X}}\left|g_{n}\left(\pi^{J^{c}}(x)\right)-f\left(\pi^{J^{c}}(x)\right)\right|<\epsilon \quad \forall n \geq N \\
\Rightarrow \quad & \sup _{x \in \mathcal{X}}\left|g_{n}^{J}(x)-f(x)\right|<\epsilon \quad \forall n \geq N \quad\left(\because \quad f\left(\pi^{J^{c}}(x)\right)=f(x)\right) \\
\Rightarrow \quad & \left\{g_{n}^{J}\right\} \mapsto f \quad \text { uniformly. }
\end{aligned}
$$

Hence $\mathcal{F}^{J}$ is dense in $\mathcal{L}_{\infty}^{J}(\mathcal{X})$.

It is also easy to see that the above proposition will hold true even if $\mathcal{L}_{\infty}^{J}(\mathcal{X})$ is replaced by any other space $\mathcal{G}$ that admits the nested structure as long as we work with the uniform metric.

(2) Since $\mathcal{F}$ is compact, for any $\epsilon>0, \exists\left\{f_{n}\right\}_{n=1}^{N_{\epsilon}} \in \mathcal{F}$ such that $\mathcal{F} \subset$ $\bigcup_{n=1}^{N_{\epsilon}} \mathbb{B}_{\|\cdot\|_{\infty}}\left(f_{n}, \epsilon\right)$ (where $\mathbb{B}_{\|\cdot\|_{\infty}}\left(f_{n}, \epsilon\right)$ is a $\|\cdot\|_{\infty}$ ball of radius $\epsilon$ with center $\left.f_{n}\right)$. We now fix $f \in \mathcal{F}^{J}$ and note that $\exists$ an equivalent class of functions $\left\{g^{f}\right\}$ in $\mathcal{F}$ such that for any two functions $g_{1}^{f}$ and $g_{2}^{f} \in\left\{g^{f}\right\}$ we have that $g_{1}^{f} \sim g_{2}^{f}$ in the sense that $g_{1}^{f} \circ \pi^{J^{c}}=g_{2}^{f} \circ \pi^{J^{c}}=f$. Fix one such $\widetilde{g}^{f} \in\left\{g^{f}\right\}$. Since $\widetilde{g}^{f} \in \mathcal{F}, \exists f_{i} \in\left\{f_{n}\right\}_{n=1}^{N_{\epsilon}}$ such that $d\left(f_{i}, \widetilde{g}^{f}\right)<\epsilon$, that is,

$$
\begin{array}{ll} 
& \sup _{x \in \mathcal{X}}\left|f_{i}(x)-\widetilde{g}^{f}(x)\right|<\epsilon \quad \Rightarrow \sup _{x \in \pi^{J^{c}}(\mathcal{X})}\left|f_{i}(x)-\widetilde{g}^{f}(x)\right|<\epsilon \\
\Rightarrow \quad & \sup _{x \in \mathcal{X}}\left|f_{i}\left(\pi^{J^{c}}(x)\right)-\widetilde{g}^{f}\left(\pi^{J^{c}}(x)\right)\right|<\epsilon \\
\Rightarrow \quad & \sup _{x \in \mathcal{X}}\left|f_{i}^{J}(x)-f(x)\right|<\epsilon \quad\left(\because \widetilde{g}^{f}\left(\pi^{J^{c}}(x)\right)=f(x)\right) \\
\Rightarrow \quad & \left\{f_{n}^{J}\right\}_{n=1}^{N_{\epsilon}} \text { forms a finite } \epsilon \text {-cover for the set } \mathcal{F}^{J} .
\end{array}
$$


Hence $\mathcal{F}^{J}$ is compact.

(3) To see (3), note that if $f_{1}, \ldots, f_{2^{n-1}}$ is an $\epsilon$-net of $\mathcal{F}$, then for any $f \in \mathcal{F}$, we have $i \in\left\{1, \ldots, 2^{n-1}\right\}$ such that $\left\|f-f_{i}\right\|_{\infty}<\epsilon$. Then,

$$
\begin{aligned}
\left\|f \circ \pi^{J^{c}}-f_{i} \circ \pi^{J^{c}}\right\|_{\infty} & =\sup _{x \in \mathcal{X}}\left|f \circ \pi^{J^{c}}(x)-f_{i} \circ \pi^{J^{c}}(x)\right|=\sup _{x \in \mathcal{X}^{J}}\left|f(x)-f_{i}(x)\right| \\
& \leq \sup _{x \in \mathcal{X}}\left|f(x)-f_{i}(x)\right|=\left\|f-f_{i}\right\|_{\infty}<\epsilon .
\end{aligned}
$$

Hence $f_{1} \circ \pi^{J^{c}}, \ldots, f_{2^{n-1}} \circ \pi^{J^{c}}$ is an $\epsilon$-net of $\mathcal{F}^{J}$.

\section{S9.3. Proof of Lemma S4.}

Proof. Note that if we define $g_{f}:=L \circ f-E_{P}(L \circ f)$, then $\mathcal{G}=\left\{g_{f}\right.$ : $f \in \mathcal{F}\}$ is a separable Carathéodory set (for a discussion on Carathéodory families of maps, refer to Definition 7.4 in SC08). To see this, first note that $\left\|g_{f}\right\|_{\infty} \leq \sup _{(x, y) \in \mathcal{X} \times \mathcal{Y}}\left|L \circ f-E_{P}(L \circ f)\right| \leq 2 B$ for $B$ defined in the statement of the Lemma. Also by assumption, $\|\cdot\|_{\mathcal{F}}$ dominates the pointwise convergence of functions (so $f_{n} \rightarrow f$ in $\|\cdot\|_{\mathcal{F}} \Rightarrow f_{n} \rightarrow f$ pointwise). Then the fact that $L$ is locally-Lipschitz continuous coupled with Lebesgue's dominated convergence theorem (since $\|L \circ f\|_{\infty} \leq B$ ) gives us the above assertion.

Now note that $E_{P}\left(g_{f}\right)=0$ and $E_{P} g_{f}^{2} \leq(2 B)^{2}=4 B^{2}$ for $B$ as before, so we can apply Talagrand's inequality as given in Theorem 7.5 of SC08 on $G$ defined as $G: \mathcal{Z}^{n} \equiv(\mathcal{X} \times \mathcal{Y})^{n} \mapsto \mathbb{R}$ such that

$$
G\left(z_{1}, \ldots, z_{n}\right):=\sup _{g_{f} \in \mathcal{G}}\left|\frac{1}{n} \sum_{j=1}^{n} g_{f}\left(z_{j}\right)\right|=\sup _{f \in \mathcal{F}}\left|\mathcal{R}_{L, D}(f)-\mathcal{R}_{L, P}(f)\right|,
$$

and hence, for $\gamma=1$ and for all $\tau>0$, we have

$$
P^{n}\left(\left\{z \in \mathcal{Z}^{n}: G(z) \geq 2 E_{P^{n}}(G)+2 B \sqrt{\frac{2 \tau}{n}}+\frac{10 B \tau}{3 n}\right\}\right) \leq e^{-\tau} .
$$

So now we need to bound the term $E_{P^{n}}(G)$ :=
$E_{P^{n}}\left\{\sup _{f \in \mathcal{F}}\left|\mathcal{R}_{L, D}(f)-\mathcal{R}_{L, P}(f)\right|\right\}$.

Defining the new Carathéodory set $\mathcal{H}$ as $\mathcal{H}=\left\{h_{f}:=L \circ f: f \in\right.$ $\mathcal{F}\}$, for a probability distribution $P$ on $\mathcal{Z} \equiv(\mathcal{X} \times \mathcal{Y})$, we can use the idea of symmetrization given in Proposition 7.10 in SC08 to bound $E_{P^{n}}\left\{\sup _{f \in \mathcal{F}}\left|\mathcal{R}_{L, D}(f)-\mathcal{R}_{L, P}(f)\right|\right\}$. We now have for all $n \geq 1$,

$$
\mathbb{E}_{D \sim P^{n}}\left\{\sup _{f \in \mathcal{F}}\left|\mathcal{R}_{L, D}(f)-\mathcal{R}_{L, P}(f)\right|\right\}=\mathbb{E}_{D \sim P^{n}} \sup _{h_{f} \in \mathcal{H}}\left|E_{P} h_{f}-E_{D} h_{f}\right|
$$




$$
\leq 2 \mathbb{E}_{D \sim P^{n}} \operatorname{Rad}_{D}(\mathcal{H}, n),
$$

where $\operatorname{Rad}_{D}(\mathcal{H}, n)$ is the $n$-th empirical Rademacher average of $\mathcal{H}$ for $D:=$ $\left\{z_{1}, \ldots, z_{n}\right\} \in \mathcal{Z}^{n}$ with respect to the Rademacher sequence $\left\{\varepsilon_{1}, \ldots, \varepsilon_{n}\right\}$ and the distribution $\nu$, which is given by $\operatorname{Rad}_{D}(\mathcal{H}, n)=E_{\nu} \sup _{h \in \mathcal{H}}\left|\frac{1}{n} \sum_{i=1}^{n} \varepsilon_{i} h\left(z_{i}\right)\right|$. So we see now that it suffices to bound $\mathbb{E}_{D \sim P^{n}} \operatorname{Rad}_{D}(\mathcal{H}, n)$.

For that we use theorem 7.16 of SC08, but before that note that the entropy bound means we have for fixed $n \geq 1$, that $\exists$ constants $a \geq 1$ and $p \in(0,1)$ such that

$$
\mathbb{E}_{D_{\mathcal{X}} \sim P_{\mathcal{X}}^{n}} e_{i}\left(\mathcal{F}, L_{\infty}\left(D_{\mathcal{X}}\right)\right) \leq a i^{-\frac{1}{2 p}}, \quad i \geq 1 .
$$

First observe that $\mathcal{H} \subset \mathcal{L}_{2}(P)$. Now since Lipschitz continuity of $L$ gives us that $\left|L\left(x, y, f_{1}(x)\right)-L\left(x, y, f_{2}(x)\right)\right|^{2} \leq c_{L}(C)^{2}\left|f_{1}(x)-f_{2}(x)\right|^{2}$, it is easy to see that

$e_{i}\left(\mathcal{H},\|\cdot\|_{L_{2}(P)}\right) \leq c_{L}(C) e_{i}\left(\mathcal{F},\|\cdot\|_{L_{2}\left(P_{\mathcal{X}}\right)}\right)$. Hence we have

$$
\begin{aligned}
\mathbb{E}_{D \sim P^{n}}\left(e_{i}\left(\mathcal{H},\|\cdot\|_{L_{2}(D)}\right)\right) & \leq c_{L}(C) \mathbb{E}_{D_{\mathcal{X}} \sim P_{\mathcal{X}}^{n}}\left(e_{i}\left(\mathcal{F},\|\cdot\|_{L_{2}\left(D_{\mathcal{X}}\right)}\right)\right) \\
& \leq c_{L}(C) \mathbb{E}_{D \mathcal{X} \sim P_{\mathcal{X}}^{n}}\left(e_{i}\left(\mathcal{F},\|\cdot\|_{L_{\infty}\left(D_{\mathcal{X}}\right)}\right)\right) \\
& \leq c_{L}(C) a i^{-\frac{1}{2 p}}
\end{aligned}
$$

Now noting that $\left\|h_{f}\right\|_{\infty} \leq B$ and $E_{P} h_{f}^{2} \leq B^{2}$ for $B$ defined as before, the conditions of Theorem 7.16 of SC08 are satisfied with $\widetilde{a}=c_{L}(C) a$ and hence we have,

$$
\mathbb{E}_{D \sim P^{n}} \operatorname{Rad}_{D}(\mathcal{H}, n) \leq \max \left\{C_{1}(p) \widetilde{a}^{p} B^{1-p} n^{-\frac{1}{2}}, C_{2}(p) \widetilde{a}^{\frac{2 p}{1+p}} B^{\frac{1-p}{1+p}} n^{-\frac{1}{1+p}}\right\}
$$

for constants $C_{1}(p), C_{2}(p)$ depending only on $p$. Hence we finally have that with probability $\geq 1-e^{-\tau}$,

$$
\begin{aligned}
& \sup _{f \in \mathcal{F}}\left|\mathcal{R}_{L, P}(f)-\mathcal{R}_{L, D}(f)\right| \leq 2 B \sqrt{\frac{2 \tau}{n}}+\frac{10 B \tau}{3 n} \\
& \quad+4 \max \left\{C_{1}(p) c_{L}(C)^{p} a^{p} B^{1-p} n^{-\frac{1}{2}}, C_{2}(p) c_{L}(C)^{\frac{2 p}{1+p}} a^{\frac{2 p}{1+p}} B^{\frac{1-p}{1+p}} n^{-\frac{1}{1+p}}\right\} .
\end{aligned}
$$

\section{S9.4. Proof of Proposition S5.}

Proof. First note that since $B \geq 1$ and $K \geq B^{p} / 4$, we have $24 K B^{1-p} \geq$ $6 B>2$. Recall the notational definitions (10) from Supplementary Section 4.1 and then see that if $a^{2 p}>\lambda^{p} n$, the inequality trivially follows from the fact that

$$
\left|\mathcal{R \mathcal { R }}_{D}^{D}\left(J_{2}, J_{1}\right)\right| \leq\left|\lambda\left\|f_{D, \lambda, H^{J_{2}}}\right\|_{H^{J_{2}}}^{2}+\mathcal{R}_{L, D}\left(f_{D, \lambda, H^{J_{2}}}\right)\right|
$$




$$
\begin{aligned}
& +\left|\lambda\left\|f_{D, \lambda, H^{J_{1}}}\right\|_{H^{J_{1}}}^{2}+\mathcal{R}_{L, D}\left(f_{D, \lambda, H^{J_{1}}}\right)\right| \\
\leq & 2 \mathcal{R}_{L, D}(0) \leq 24 K B^{1-p}\left(\frac{a^{2 p}}{\lambda^{p} n}\right)^{\frac{1}{2}},
\end{aligned}
$$

since $\mathcal{R}_{L, D}(0) \leq 1$. Hence we assume from here on that $a^{2 p} \leq \lambda^{p} n$. Now observe that since $H$ is separable, from Lemma S2 in Section S1 we have that the $H^{J}$ S are also separable. Hence from Lemma 6.23 of SC08 we have that the KMs produced by these RKHSs are measurable.

Now note that $L(x, y, 0) \leq 1 \Rightarrow$ for any distribution $Q$ on $\mathcal{X} \times \mathcal{Y}$, we have that $\mathcal{R}_{L, Q}(0) \leq 1$. Since, $\inf _{f \in H^{J}} \lambda\|f\|_{H^{J}}^{2}+\mathcal{R}_{L, Q}(f) \leq \mathcal{R}_{L, Q}(0)$, we have that $\left\|f_{Q, \lambda, H^{J}}\right\|_{H^{J}} \leq \sqrt{\frac{\mathcal{R}_{L, Q}(0)}{\lambda}}$. Now since by Lemma 4.23 of SC08 $\|f\|_{\infty} \leq$ $\|k\|_{\infty}\|f\|_{H^{J}}$ for all $f \in H^{J}$, we have that $\left\|f_{Q, \lambda, H^{J}}\right\|_{\infty} \leq\left\|f_{Q, \lambda, H^{J}}\right\|_{H^{J}} \leq$ $\lambda^{-1 / 2}$. So, consequently, for every distribution $Q$ on $\mathcal{X} \times \mathcal{Y}$, we have

$$
\left|\mathcal{R}_{L, P}\left(f_{Q, \lambda, H^{J}}\right)-\mathcal{R}_{L, D}\left(f_{Q, \lambda, H^{J}}\right)\right| \leq \sup _{\|f\|_{H^{J}} \leq \lambda^{-1 / 2}}\left|\mathcal{R}_{L, P}(f)-\mathcal{R}_{L, D}(f)\right| .
$$

Now since from (A1) in Section 3.3, $\mathcal{R}^{*}{ }_{L, P, H^{J_{1}}}=\mathcal{R}^{*}{ }_{L, P, H^{J_{2}}}=\mathcal{R}^{*}{ }_{L, P, H}$, we have

$$
\begin{aligned}
& \left|\mathcal{R R}_{D}^{D}\left(J_{2}, J_{1}\right)\right| \leq\left|\mathcal{R}_{P}^{D}\left(J_{2}\right)\right|+\left|\mathcal{R R}_{P}^{D}\left(J_{1}\right)\right|+\left|\mathcal{R}_{L, D}\left(f_{D, \lambda, H^{J_{2}}}\right)-\mathcal{R}_{L, P}\left(f_{D, \lambda, H^{J_{2}}}\right)\right| \\
& +\left|\mathcal{R}_{L, D}\left(f_{D, \lambda, H^{J_{1}}}\right)-\mathcal{R}_{L, P}\left(f_{D, \lambda, H^{J_{1}}}\right)\right| .
\end{aligned}
$$

Noting that $\mathcal{R R}_{P}^{D}(J) \geq 0$, we have from (6.18) of SC08

$$
\begin{aligned}
& \left|\mathcal{R R}_{P}^{D}(J)\right| \leq A_{2}^{J}(\lambda)+\mathcal{R}_{L, P}\left(f_{D, \lambda, H^{J}}\right)-\mathcal{R}_{L, D}\left(f_{D, \lambda, H^{J}}\right) \\
& \quad+\mathcal{R}_{L, D}\left(f_{P, \lambda, H^{J}}\right)-\mathcal{R}_{L, P}\left(f_{P, \lambda, H^{J}}\right) \leq A_{2}^{J}(\lambda)+2 \sup _{\|f\|_{H^{J}} \leq \lambda^{-1 / 2}}\left|\mathcal{R}_{L, P}(f)-\mathcal{R}_{L, D}(f)\right| .
\end{aligned}
$$

From (11) and (12) and the fact that $J_{1}, J_{2} \in \tilde{\mathcal{J}}$ such that $J_{1} \subseteq J_{2} \subseteq J_{*}$, we have that

$$
\begin{aligned}
& \left|\mathcal{R R}_{D}^{D}\left(J_{2}, J_{1}\right)\right| \leq 3 \sup _{\|f\|_{H^{J_{1}} \leq \lambda^{-1 / 2}}\left|\mathcal{R}_{L, P}(f)-\mathcal{R}_{L, D}(f)\right|} \\
& \quad+3 \sup _{\|f\|_{H^{J}} \leq \lambda^{-1 / 2}}\left|\mathcal{R}_{L, P}(f)-\mathcal{R}_{L, D}(f)\right|+A_{2}{ }^{J_{1}}(\lambda)+A_{2}{ }^{J_{2}}(\lambda) .
\end{aligned}
$$

First note that for $f \in \lambda^{-1 / 2} B_{H^{J}}$ and $B:=c_{L}\left(\lambda^{-1 / 2}\right) \lambda^{-1 / 2}+1$, we have $|L(x, y, f(x))| \leq|L(x, y, f(x))-L(x, y, 0)|+L(x, y, 0) \leq B$ for all $(x, y) \in \mathcal{X} \times \mathcal{Y}$. Also note that the entropy bound assumption implies that $E_{D_{\mathcal{X}} \sim P_{\mathcal{X}}^{n}}\left(e_{i}\left(\lambda^{-1 / 2} B_{H},\|\cdot\|_{L_{\infty}\left(D_{\mathcal{X}}\right)}\right)\right) \leq \lambda^{-1 / 2} a i^{-\frac{1}{2 p}}$. 
Now note from Lemma S2 in Supplementary Section S1 that the conditions of Lemma S4 are satisfied for $\mathcal{F}:=\lambda^{-1 / 2} B_{H^{J}},\|\cdot\|_{\mathcal{F}}:=\|\cdot\|_{H^{J}}$, $C:=\lambda^{-1 / 2}$ and $B:=c_{L}\left(\lambda^{-1 / 2}\right) \lambda^{-1 / 2}+1$ for each of the RKHS classes $H^{J}$. Also since $a^{2 p} \leq \lambda^{p} n$ and $B \geq 1$, we have $\left(\frac{a^{2 p}}{\lambda^{p} n}\right)^{1 / 2} \geq\left(\frac{a^{2 p}}{\lambda^{p} n}\right)^{1 /(p+1)}$ and $B^{1-p} \geq B^{\frac{1-p}{1+p}}$ for $p \in(0,1)$. Hence we have our assertion.

\section{GLOSSARY}

$\mathcal{L}_{\infty} \quad$ The set of all bounded measurable functions on a given space, that is, $\mathcal{L}_{\infty}(\mathcal{X})=\left\{f: \mathcal{X} \mapsto \mathbb{R}, f\right.$ measurable and $\left.\|f\|_{\infty}<\infty\right\}$. 1,19

$\pi \quad$ The projection map. See Definition 1. 1, 2

$e_{i} \quad$ The $i$-th entropy number for a given metric space, for mathematical definition see (5). 1, 3, 4, 23, 24

$k \quad k(x, y)=\langle\phi(x), \phi(x)\rangle_{H}$, the kernel function associated with a given reproducing kernel Hilbert space (RKHS). 2, 4, 12, 24

$L \quad L: \mathcal{X} \times \mathcal{Y} \times \mathbb{R} \mapsto[0, \infty]$ is a convex, locally Lipschitz continuous and measurable loss function. $3,4,22,23,24,25$

$\mathbb{E}_{D_{\mathcal{X}} \sim P_{\mathcal{X}}^{n}}$ The expectation w.r.t. $P_{\mathcal{X}}^{n}$ for data $D_{\mathcal{X}} \equiv\left\{\mathcal{X}_{1}, \ldots, \mathcal{X}_{n}\right\}$ being i.i.d. copies of $\mathcal{X} \sim P_{\mathcal{X}}$. Similarly $\mathbb{E}_{D \sim P^{n}}$ for the joint measure $P$ and full data D. 3, 4, 22, 23

$L_{\infty}\left(D_{\mathcal{X}}\right) \quad$ The space of equivalence classes of all bounded measurable functions w.r.t. the measure $D_{\mathcal{X}} 3,4,23,24$

$\mathcal{R}_{L, P} \quad \mathcal{R}_{L, P}(f)=E_{P}[L(X, Y, f(X)] .3,4,19,22,23,24$

$\mathcal{R}_{L, D} \quad \mathcal{R}_{L, D}(f)=\frac{1}{n} \sum_{i=1}^{n} L\left(X_{i}, Y_{i}, f\left(X_{i}\right)\right.$ for a given data $D$ of size $n$. $3,4,22,23,24$

$H \quad$ A separable RKHS of the measurable kernel $k$ on $\mathcal{X}$. 4, 19, 24

reg Implies regularized risk, for example $\mathcal{R}_{L, Q, \mathcal{F}}^{\mathrm{reg}, \lambda}(f)=\lambda\|f\|_{\mathcal{F}}^{2}+$ $\mathcal{R}_{L, Q}(f) .4,5,19$

$A_{2} \quad$ The approximation error for a given optimization space and a $\lambda$, for example, $A_{2}^{J}(\lambda)=\mathcal{R}_{L, P, H^{J}}^{\mathrm{reg}, \lambda}\left(f_{P, \lambda, H^{J}}\right)-\mathcal{R}_{L, P, H^{J}}^{*} \cdot 4,5,18,19$, 20,24

$\mathcal{R}^{*} \quad$ The minimal risk attained within a space, for example, $\mathcal{R}_{L, P, \mathcal{F}}^{*}=$ $\mathcal{R}_{L, P}\left(f_{P, \mathcal{F}}\right) .4,5,19,24$

\section{REFERENCES}

Guyon, I., Weston, J., Barnhill, S. and VApnik, V. (2002). Gene selection for cancer classification using support vector machines. Machine Learning 46 389-422.

Hastie, T., Tibshirani, R. and Friedman, J. (2009). The elements of statistical learning 2nd edition. Springer. 
Iqbal, M. J., FAye, I., SAmir, B. B. and Md SAid, A. (2014). Efficient feature selection and classification of protein sequence data in bioinformatics. The Scientific World Journal 2014.

Leslie, C. S., Eskin, E., Cohen, A., Weston, J. and Noble, W. S. (2004). Mismatch string kernels for discriminative protein classification. Bioinformatics 20 467-476.

PAulsen, V. I. (2009). An introduction to the theory of reproducing kernel Hilbert spaces. Unpublished notes.

Rabiner, L. R. and Schafer, R. W. (1978). Digital processing of speech signals. PrenticeHall.

Steinwart, I. and Chirstmann, A. (2008). Support vector machines. Springer.

Steinwart, I. and Scovel, C. (2007). Fast rates for support vector machines using Gaussian kernels. Annals of Statistics 35 575-607.

Tsanas, A. T., Little, M. A., McSharry, P. E. and Ramig, L. O. (2010). Accurate telemonitoring of Parkinson's disease progression by noninvasive speech tests. IEEE transactions on Biomedical Engineering $\mathbf{5 7} 884-893$. 